\title{
TRACES ON THE SKLYANIN ALGEBRA AND CORRELATION FUNCTIONS OF THE EIGHT-VERTEX MODEL
}

\author{
H. BOOS, M. JIMBO, T. MIWA, F. SMIRNOV AND Y. TAKEYAMA
}

\begin{abstract}
We propose a conjectural formula for correlation functions of the Z-invariant (inhomogeneous) eight-vertex model. We refer to this conjecture as Ansatz. It states that correlation functions are linear combinations of products of three transcendental functions, with theta functions and derivatives as coefficients. The transcendental functions are essentially logarithmic derivatives of the partition function per site. The coefficients are given in terms of a linear functional $\operatorname{Tr}_{\lambda}$ on the Sklyanin algebra, which interpolates the usual trace on finite dimensional representations. We establish the existence of $\operatorname{Tr}_{\lambda}$ and discuss the connection to the geometry of the classical limit. We also conjecture that the Ansatz satisfies the reduced qKZ equation. As a non-trivial example of the Ansatz, we present a new formula for the next-nearest neighbor correlation functions.
\end{abstract}

\section{INTRODUCTION}

Exact description of correlation functions and their analysis is one of the central problems of integrable lattice models. Significant progress has been made over the last decade toward this goal. In the study of correlation functions, a basic role is played by a multiple integral representation, first found for the archetypical example of the spin 1/2-XXZ chain [17, 15, 20]. Subsequently it has been generalized in several directions, to incorporate an external field [20], unequal time [19], non-zero temperature [13] and finite chains [18. Earlier in the literature, extension to elliptic models has also been pursued. The free field construction used in the XXZ model was extended in 24] to the SOS models, resulting in an integral formula for correlation functions of the ABF model. In [25] an integral formula was obtained for the eightvertex model by mapping the problem to the SOS counterpart. A novel free field representation of the eight-vertex model is being developed in [33, 34.

Recent studies have revealed another aspect of these integrals. Through examples at short distance, it has been observed in the case of the homogeneous XXX chain that the relevant integrals can be evaluated in terms of the Riemann zeta function at odd integers with rational coefficients [5]. Similar calculations have been performed for the XXZ chain [23, 37. This phenomenon was explained later through a duality between the qKZ equations of level 0 and level -4 [6, 7]. Motivated by these works, we have established in our previous papers [3, 4] an algebraic representation (in the

Date: August 19, 2018. 
sense no integrals are involved) for general correlation functions of the inhomogeneous six-vertex model and its degeneration ${ }^{1}$. The aim of the present paper is to continue our study and examine the eight-vertex model.

We formulate a conjectural formula for correlation functions (the Ansatz) along the same line with the six-vertex case. Consider the eight-vertex model where each column $i$ (resp. row $j$ ) carries an independent spectral parameter $t_{i}$ (resp. 0). The object of our interest is the matrix

$$
\begin{aligned}
& h_{n}\left(t_{1}, \cdots, t_{n}\right) \\
& \quad=\frac{1}{2^{n}} \sum_{\alpha_{1}, \cdots, \alpha_{n}=0}^{3} \varepsilon_{a_{1}} \cdots \varepsilon_{a_{n}}\left\langle\sigma_{1}^{\alpha_{1}} \cdots \sigma_{n}^{\alpha_{n}}\right\rangle\left(\sigma^{\alpha_{1}} \otimes \cdots \otimes \sigma^{\alpha_{n}}\right)^{T} \quad \in \operatorname{End}\left(\left(\mathbb{C}^{2}\right)^{\otimes n}\right)
\end{aligned}
$$

where $\langle\cdots\rangle$ denotes the ground state average in the thermodynamic limit, $\sigma^{0}=1, \sigma^{a}$ $(1 \leq a \leq 3)$ are the Pauli matrices, and $T$ stands for the matrix transpose. Regard $h_{n}$ as a vector via the identification $\operatorname{End}\left(\left(\mathbb{C}^{2}\right)^{\otimes n}\right) \simeq\left(\mathbb{C}^{2}\right)^{\otimes 2 n}$, and let $\mathbf{s}_{n}$ denote the vector corresponding to the identity. Our Ansatz is that $h_{n}$ can be represented in the form

$$
h_{n}\left(t_{1}, \cdots, t_{n}\right)=2^{-n} \exp \left(\sum_{i<j} \sum_{a=1}^{3} \omega_{a}\left(t_{i j}\right) \widehat{X}_{a, n}^{(i, j)}\left(t_{1}, \cdots, t_{n}\right)\right) \mathbf{s}_{n} .
$$

Here $\omega_{a}(t)$ are scalar functions given explicitly in terms of the partition function per site (see (2.32) below). The matrices $\widehat{X}_{a, n}^{(i, j)}$ are expressible by theta functions and derivatives. Leaving the details to Section [2.4, let us comment on the latter.

In the six-vertex case, $\widehat{X}_{a, n}^{(i, j)}$ are defined in terms of a 'trace' of a monodromy matrix. Here 'trace' means the unique linear functional

$$
\operatorname{Tr}_{\lambda}: U_{q}\left(\mathfrak{s l}_{2}\right) \longrightarrow \mathbb{C}\left[q^{ \pm \lambda}\right] \oplus \lambda \mathbb{C}\left[q^{ \pm \lambda}\right]
$$

which for $\lambda \in \mathbb{Z}_{\geq 0}$ reduces to the usual trace on the $\lambda$-dimensional irreducible representation of $\bar{U}_{q}\left(\mathfrak{s l}_{2}\right)$. In the eight-vertex case, we need an analogous functional $\operatorname{Tr}_{\lambda}$, defined on the Sklyanin algebra and taking values in the space of entire functions involving $\lambda$, theta functions and derivatives. Compared with the trigonometric case, the existence of $\operatorname{Tr}_{\lambda}$ is more difficult to establish. We do that by considering the classical limit and showing that, for generic values of the structure constants, the computation of the trace of an arbitrary monomial can be reduced to that of seven basic monomials. We have also compared our formula for $\operatorname{Tr}_{\lambda}$ with the results by K. Fabricius and B. McCoy [8] for $\lambda=3,4,5$. In the classical limit, the Sklyanin algebra becomes the algebra of regular functions on an algebraic surface in $\mathbb{C}^{4}$, which turns out to be a smoothing of a simple-elliptic singularity of K. Saito [29]. The reduction of the trace is closely connected with the de Rham cohomology of this surface (see Appendix $\mathrm{A}$ ). Although we do not use Saito's results for our immediate purposes, we find this connection intriguing.

\footnotetext{
${ }^{1}$ Correlation functions of the XXZ and XXX chains are given in the limit where all the inhomogeneity parameters are chosen to be the same. However we have not succeeded in performing this homogeneous limit.
} 
In the trigonometric case, it was shown [4] that the functions given by the above Ansatz satisfies the reduced qKZ equation. The steps of the proof carry over straightforwardly to the elliptic case, except for one property (the Cancellation identity). Unfortunately we have not succeeded in proving this last relation. It remains an open question to show that our Ansatz in the elliptic case satisfies the reduced qKZ equation.

To check the validity of the Ansatz, we examine the simplest case $n=2$. In this case an exact answer for the homogeneous chain is obtained as derivatives of the ground state energy of the spin-chain Hamiltonian. Our formula matches with it. We also present an explicit formula for the correlators with $n=3$. It agrees well numerically with the known integral formulas of [25, 26].

The plan of the paper is as follows. In Section 2, we introduce our notation and formulate the Ansatz for correlation functions. In Section 3, we discuss the validity of the Cancellation Identity and give arguments in its favor. Section 4 is devoted to the examples for correlators of the nearest and the next nearest neighbor spins. We also discuss briefly the trigonometric limit. In Appendix A we prove the existence of the trace functional. As was mentioned above, the classical limit of the Sklyanin algebra is related to an affine algebraic surface, and the the trace functional tends to an integral over a certain cycle on it. We explain the connection between this picture and K. Saito's theory. In Appendix B, we give an explicit description of the integration cycles. Appendix $\mathrm{C}$ contains technical Lemmas about the trace. Finally in Appendix $\mathrm{D}$ we discuss the transformation properties of the matrices $\widehat{X}_{a, n}^{(i, j)}$.

\section{AnsATZ FOR CORRELATION FUNCTIONS}

In this section we introduce our notation and formulate the Ansatz for correlation functions of an inhomogeneous eight-vertex model, following the scheme developed in [4].

2.1. $R$ matrix. We consider an elliptic $R$ matrix depending on three complex parameters $t, \eta, \tau$. We assume $\operatorname{Im} \tau>0$ and $\eta \notin \mathbb{Q}+\mathbb{Q} \tau^{2}$. We will normally regard $\eta, \tau$ as fixed constants and suppress them from the notation. Let $\theta_{\alpha}(t)=\theta_{\alpha}(t \mid \tau)$ $\left(0 \leq \alpha \leq 4, \theta_{4}(t)=\theta_{0}(t)\right)$ denote the Jacobi elliptic theta functions associated with the lattice $\mathbb{Z}+\mathbb{Z} \tau$ [14]. We set

$$
[t]:=\frac{\theta_{1}(2 t)}{\theta_{1}(2 \eta)}
$$

The $R$ matrix is given by

$$
\begin{aligned}
& R(t):=\rho(t) \frac{r(t)}{[t+\eta]} \in \operatorname{End}(V \otimes V), \\
& r(t):=\frac{1}{2} \sum_{\alpha=0}^{3} \frac{\theta_{\alpha+1}(2 t+\eta)}{\theta_{\alpha+1}(\eta)} \sigma^{\alpha} \otimes \sigma^{\alpha},
\end{aligned}
$$

where $V=\mathbb{C} v_{+} \oplus \mathbb{C} v_{-}$.

\footnotetext{
${ }^{2}$ Later on we also assume that $\eta$ is generic
} 
The matrix $r(t)=r_{12}(t)$ is the unique entire function satisfying

$$
\begin{aligned}
& r_{12}(0)=P_{12}, \\
& \sigma_{1}^{a} \sigma_{2}^{a} r_{12}(t)=r_{12}(t) \sigma_{1}^{a} \sigma_{2}^{a} \quad(a=1,2,3), \\
& r_{12}\left(t+\frac{1}{2}\right)=-\sigma_{1}^{1} r_{12}(t) \sigma_{1}^{1}, \\
& r_{12}\left(t+\frac{\tau}{2}\right)=-\sigma_{1}^{3} r_{12}(t) \sigma_{1}^{3} \times e^{-2 \pi i(2 t+\eta+\tau / 2)} .
\end{aligned}
$$

Here $P \in \operatorname{End}(V \otimes V)$ signifies the transposition $P u \otimes v=v \otimes u$. As is customary, the suffix of a matrix indicates the tensor component on which it acts non-trivially, e.g. $\sigma_{1}^{\alpha}=\sigma^{\alpha} \otimes 1, \sigma_{2}^{\alpha}=1 \otimes \sigma^{\alpha}$.

The normalizing factor $\rho(t)$ is chosen to ensure that the partition function per site of the corresponding eight-vertex model equals to 1 . Its explicit formula depends on the regime under consideration, and will be given later in (2.28),(2.29). In each case it satisfies

$$
\rho(t) \rho(-t)=1, \quad \rho(t) \rho(t-\eta)=\frac{[t]}{[\eta-t]},
$$

We will often write $t_{i j}=t_{i}-t_{j}$. The basic properties of $R(t)$ are the Yang-Baxter equation

$$
R_{12}\left(t_{12}\right) R_{13}\left(t_{13}\right) R_{23}\left(t_{23}\right)=R_{23}\left(t_{23}\right) R_{13}\left(t_{13}\right) R_{12}\left(t_{12}\right),
$$

and

$$
\begin{aligned}
& R(t)=P R(t) P \\
& R(-\eta)=-2 \mathcal{P}^{-} \\
& R_{12}(t) R_{21}(-t)=1 \\
& R_{12}(t) \mathcal{P}_{23}^{-}=-R_{13}(-t-\eta) \mathcal{P}_{23}^{-} .
\end{aligned}
$$

In (2.5), $\mathcal{P}^{-}=(1-P) / 2$ denotes the projection onto the one-dimensional subspace spanned by

$$
s:=v_{+} \otimes v_{-}-v_{-} \otimes v_{+} \quad \in V \otimes V
$$

We will use also

$$
\check{R}(t)=P R(t)
$$

2.2. Sklyanin algebra. Along with the $R$ matrix, we will need the $L$ operator whose entries are generators of the Sklyanin algebra [35, 36].

Recall that the Sklyanin algebra $\mathcal{A}$ is an associative unital $\mathbb{C}$-algebra defined through four generators $S_{\alpha}(\alpha=0,1,2,3)$ and quadratic relations

$$
\begin{aligned}
& {\left[S_{0}, S_{a}\right]=i J_{b c}\left(S_{b} S_{c}+S_{c} S_{b}\right),} \\
& {\left[S_{b}, S_{c}\right]=i\left(S_{0} S_{a}+S_{a} S_{0}\right),}
\end{aligned}
$$


where $(a, b, c)$ runs over cyclic permutations of $(1,2,3)$. The $J_{b c}$ are the structure constants given by

$$
\begin{aligned}
& J_{b c}=-\frac{J_{b}-J_{c}}{J_{a}}=\varepsilon_{a} \frac{\theta_{1}(\eta)^{2} \theta_{a+1}(\eta)^{2}}{\theta_{b+1}(\eta)^{2} \theta_{c+1}(\eta)^{2}} \\
& J_{a}=\frac{\theta_{a+1}(2 \eta) \theta_{a+1}}{\theta_{a+1}(\eta)^{2}}
\end{aligned}
$$

where

$$
\varepsilon_{2}=-1, \quad \varepsilon_{\alpha}=1(\alpha \neq 2) .
$$

Here and after, theta functions without arguments stand for the theta zero values, $\theta_{a}=\theta_{a}(0)$ and $\theta_{1}^{\prime}=\theta_{1}^{\prime}(0)$.

Since the defining relations are homogeneous, $\mathcal{A}$ is a $\mathbb{Z}_{\geq 0}$-graded algebra, $\mathcal{A}=$ $\oplus_{n \geq 0} \mathcal{A}_{n}$, where the generators $S_{a}$ all belong to $\mathcal{A}_{1}$. We have also a $\mathbb{Z}_{2} \times \mathbb{Z}_{2}$-grading, $\mathcal{A}=\oplus_{(m, n) \in \mathbb{Z}_{2} \times \mathbb{Z}_{2}} \mathcal{A}^{(m, n)}$, defined by the assignment $S_{\alpha} \in \mathcal{A}^{\bar{\alpha}}$, where

$$
\overline{0}=(0,0), \overline{1}=(1,0), \overline{2}=(1,1), \overline{3}=(0,1) \quad \in \mathbb{Z}_{2} \times \mathbb{Z}_{2} .
$$

To make distinction, the $\mathbb{Z}_{\geq 0}$-grading and the $\mathbb{Z}_{2} \times \mathbb{Z}_{2}$-grading will be referred to as 'degree' and 'color', respectively. Thus $S_{\alpha}$ has degree 1 and color $\bar{\alpha}$.

There are two central elements of degree 2 and color $\overline{0}$,

$$
K_{0}:=\sum_{\alpha=0}^{3} S_{\alpha}^{2}, \quad K_{2}:=\sum_{a=1}^{3} J_{a} S_{a}^{2}
$$

We call them Casimir elements.

Introduce the generating function ( $L$ operator)

$$
L(t):=\frac{1}{2} \sum_{\alpha=0}^{3} \frac{\theta_{\alpha+1}(2 t+\eta)}{\theta_{\alpha+1}(\eta)} S_{\alpha} \otimes \sigma^{\alpha} \quad \in \mathcal{A} \otimes \operatorname{End}(V) .
$$

The defining relations (2.8), (2.9) are equivalent to

$$
R_{12}(t-s) L_{1}(t) L_{2}(s)=L_{2}(s) L_{1}(t) R_{12}(t-s) .
$$

From (2.13) we have

$$
L_{1}\left(\frac{t}{2}\right) L_{2}\left(\frac{t}{2}-\eta\right) \mathcal{P}_{12}^{-}=-\frac{1}{4}\left(\frac{\theta_{1}(\eta-t) \theta_{1}(\eta+t)}{\theta_{1}(\eta)^{2}} K_{0}+\frac{\theta_{1}(t)^{2}}{\theta_{1}(\eta)^{2}} K_{2}\right) \mathcal{P}_{12}^{-}
$$

We will be concerned with representations in series (a) of [36, which are analogs of finite-dimensional irreducible representations of $\mathfrak{s l}_{2}$. For each non-negative integer $k$, let $\mathcal{V}^{(k)}$ denote the vector space of entire functions $f(u)$ with the properties

$$
f(u+1)=f(u)=f(-u), \quad f(u+\tau)=e^{-2 \pi i k(2 u+\tau)} f(u) .
$$


We have $\operatorname{dim} \mathcal{V}^{(k)}=k+1$. The following formula defines a representation $\pi^{(k)}: \mathcal{A} \rightarrow$ $\operatorname{End}\left(\mathcal{V}^{(k)}\right)[36]{ }^{3}$

$$
\begin{aligned}
& \left(\pi^{(k)}\left(S_{\alpha}\right) f\right)(u) \\
& \quad=\frac{\sqrt{\varepsilon_{\alpha}} \theta_{\alpha+1}(\eta)}{\theta_{1}(2 \eta) \theta_{1}(2 u)}\left(\theta_{\alpha+1}(2 u-k \eta) e^{\eta \partial_{u}}-\theta_{\alpha+1}(-2 u-k \eta) e^{-\eta \partial_{u}}\right) f(u) .
\end{aligned}
$$

Here $\sqrt{\varepsilon_{2}}=i, \sqrt{\varepsilon_{\alpha}}=1(\alpha \neq 2)$, and $\left(e^{ \pm \eta \partial_{u}} f\right)(u)=f(u \pm \eta)$. In particular, if $k=1$, then in an appropriate basis we have

$$
\pi^{(1)}\left(S_{\alpha}\right)=\sigma^{\alpha}, \quad\left(\pi^{(1)} \otimes \mathrm{id}\right) L(t)=r(t)
$$

On $\mathcal{V}^{(k)}$, the Casimir elements $K_{0}, K_{2}$ act as scalars $K_{0}(k+1), K_{2}(k+1)$ respectively, where

$$
K_{0}(\lambda)=4 \frac{\theta_{1}(\lambda \eta)^{2}}{\theta_{1}(2 \eta)^{2}}, \quad K_{2}(\lambda)=4 \frac{\theta_{1}(\lambda \eta+\eta) \theta_{1}(\lambda \eta-\eta)}{\theta_{1}(2 \eta)^{2}}
$$

2.3. The functional $\operatorname{Tr}_{\lambda}$. In order to formulate the Ansatz, we need to consider the $\operatorname{trace}_{\operatorname{tr}_{\mathcal{V}}(k)} \pi^{(k)}(A)$ of an element $A \in \mathcal{A}$ as a function of the dimension $k+1$. The precise meaning is as follows.

For each $A \in \mathcal{A}$ one can assign a unique entire function $\operatorname{Tr}_{\lambda} A$ in $\lambda$ with the following properties:

(i) $\left.\operatorname{Tr}_{\lambda} A\right|_{\lambda=k+1}=\operatorname{tr}_{\mathcal{V}^{(k)}} \pi^{(k)}(A)$ holds for all $k \in \mathbb{Z}_{\geq 0}$,

(ii) If $A \in \mathcal{A}_{n}, \operatorname{Tr}_{\lambda} A$ has the functional form

$$
\operatorname{Tr}_{\frac{t}{\eta}} A=\theta_{1}(t)^{n} \times \begin{cases}g_{A, 0}(t) & (n: \text { odd }), \\ g_{A, 1}(t)-\frac{t}{\eta} g_{A, 2}(t) & (n: \text { even }),\end{cases}
$$

where $g_{A, 0}(t), g_{A, 2}(t)$ and $g_{A, 3}(t):=g_{A, 1}(t+\tau)-g_{A, 1}(t)$ are elliptic functions with periods $1, \tau$. In addition, $g_{A, 1}(t+1)=g_{A, 1}(t)$.

For example,

$$
\begin{aligned}
& \operatorname{Tr}_{\lambda} 1=\lambda \\
& \operatorname{Tr}_{\lambda} S_{\alpha}=2 \delta_{\alpha 0} \frac{\theta_{1}(\lambda \eta)}{\theta_{1}(2 \eta)} \\
& \operatorname{Tr}_{\lambda} S_{\alpha}^{2}=\frac{2}{\theta_{1}^{\prime} \theta_{1}(2 \eta)^{3}}\left(F_{\alpha 1}(\lambda \eta)-\lambda F_{\alpha 2}(\lambda \eta)\right)
\end{aligned}
$$

where

$$
\begin{aligned}
& F_{\alpha 1}(t)=\varepsilon_{\alpha} \theta_{\alpha+1}(\eta)^{2} \frac{\partial}{\partial t}\left(\theta_{\alpha+1}(t+\eta) \theta_{\alpha+1}(t-\eta)\right) \\
& F_{\alpha 2}(t)=\varepsilon_{\alpha} \theta_{\alpha+1}(\eta)^{2} \frac{\partial}{\partial \eta}\left(\theta_{\alpha+1}(t+\eta) \theta_{\alpha+1}(t-\eta)\right) .
\end{aligned}
$$

For reference we set

$$
F_{\alpha 3}(t)=\varepsilon_{\alpha} \theta_{\alpha+1}(\eta)^{2} \theta_{\alpha+1}(t+\eta) \theta_{\alpha+1}(t-\eta) .
$$

\footnotetext{
${ }^{3}$ We have modified eq.(6) of [36] by a factor $\theta_{1}(2 \eta)$.
} 
$\operatorname{Tr}_{\lambda}$ satisfies also

$$
\begin{aligned}
& \operatorname{Tr}_{\lambda}(A B)=\operatorname{Tr}_{\lambda}(B A), \\
& \operatorname{Tr}_{\lambda}\left(K_{i} A\right)=K_{i}(\lambda) \operatorname{Tr}_{\lambda}(A) \quad(i=0,2), \\
& \operatorname{Tr}_{\lambda} A=0 \quad\left(A \in \mathcal{A}^{(m, n)},(m, n) \neq(0,0)\right) .
\end{aligned}
$$

The derivation of (2.27) as well as (2.20), (2.21) is sketched in Appendix C] In Appendix $\mathrm{A}$ we show that, for generic $\eta$, any element $A \in \mathcal{A} / \sum_{\alpha=0}^{3}\left[S_{\alpha}, \mathcal{A}\right]$ can be written as a $\mathbb{C}\left[K_{0}, K_{2}\right]$-linear combination of seven monomials: $1, S_{0}, S_{1}, S_{2}, S_{3}, S_{0}^{2}, S_{3}^{2}$. Hence $\operatorname{Tr}_{\lambda} A$ is completely determined by the property (2.25)-(2.27) along with (2.19) - 2.21). However an effective algorithm for the reduction is not known to us. The situation is in sharp contrast to the trigonometric case, where a simple recursive procedure for calculating $\operatorname{Tr}_{\lambda}$ is available (see [4]). It would be useful if one can find a more direct expression for the trace using $Q$-operators, as is done for the XXZ model in 22 .

In this connection, notice that by the above rule, $\operatorname{Tr}_{\lambda} A$ has a simpler structure for elements of odd degree than those of even degree, since in the former case it is a polynomial of $\theta_{1}(t+c)(c \in \mathbb{C})$ without involving derivatives. The subtle difference between finite spin chains with odd length and those with even length has been noticed in the context of $Q$-operators [27, 9, 21.

2.4. The Ansatz. Consider an inhomogeneous eight vertex model, where each column $i$ (resp. row $j$ ) carries a spectral parameter $t_{i}$ (resp. 0). The Boltzmann weights are given by the entries $R_{\varepsilon_{1}, \varepsilon_{2}}^{\varepsilon_{1}^{\prime}, \varepsilon_{2}^{\prime}}\left(t_{i}\right)$ of the $R$ matrix (2.1). We choose the normalizing factor $\rho(t)$ in (2.1) in accordance with the two regimes

(i) $\eta, t \in i \mathbb{R},-i \eta>0$ (disordered regime)

(ii) $\eta, t \in \mathbb{R}, \eta<0$ (ordered regime)

In the disordered regime, $\rho(t)=\rho^{d i s}(t)$ is given by [1]

$$
\begin{aligned}
& \rho^{d i s}(t):=e^{-2 \pi i t} \times \frac{\gamma(2 \eta-2 t)}{\gamma(2 \eta+2 t)} \frac{\gamma(4 \eta+2 t)}{\gamma(4 \eta-2 t)}, \\
& \gamma(u)=\Gamma(u, 4 \eta, \tau)
\end{aligned}
$$

where

$$
\Gamma(u, \sigma, \tau):=\prod_{j, k=0}^{\infty} \frac{1-e^{2 \pi i((j+1) \tau+(k+1) \sigma-u)}}{1-e^{2 \pi i(j \tau+k \sigma+u)}}
$$

is the elliptic gamma function [28]. In the ordered regime, the formula for $\rho(t)=$ $\rho^{\text {ord }}(t)$ is changed to

$$
\rho^{\text {ord }}(t)=e^{-4 \pi i \eta t / \tau} \rho^{\prime}\left(t^{\prime}\right)
$$

where $\rho^{\prime}\left(t^{\prime}\right)$ is given by the right hand side of (2.28) with $t, \eta, \tau$ being replaced by

$$
t^{\prime}=\frac{t}{\tau}, \quad \eta^{\prime}=\frac{\eta}{\tau}, \quad \tau^{\prime}=-\frac{1}{\tau},
$$

respectively. 
By correlation functions we mean the ground state averages $\left\langle\sigma_{1}^{\alpha_{1}} \cdots \sigma_{n}^{\alpha_{n}}\right\rangle$ of a product of spin operators on consecutive columns $1, \ldots, n$ on a same row of the lattice. The thermodynamic limit is assumed. We arrange them into a matrix

$$
\begin{aligned}
& h_{n}\left(t_{1}, \cdots, t_{n}\right) \\
& \quad=\frac{1}{2^{n}} \sum_{\alpha_{1}, \cdots, \alpha_{n}=0}^{3}\left\langle\sigma_{1}^{\alpha_{1}} \cdots \sigma_{n}^{\alpha_{n}}\right\rangle\left(\sigma^{\alpha_{1}}\right)^{T} \otimes \cdots \otimes\left(\sigma^{\alpha_{n}}\right)^{T} \quad \in \operatorname{End}\left(V^{\otimes n}\right),
\end{aligned}
$$

where $\left(\sigma_{i}^{\alpha}\right)^{T}=\varepsilon_{\alpha} \sigma_{i}^{\alpha}$ stands for the transposed matrix. Because of the 'Z-invariance' [2], it does not depend on $t_{i}$ with $i<1$ or $i>n$. When $t_{1}=\cdots=t_{n}$, each $\left\langle\sigma_{1}^{\alpha_{1}} \cdots \sigma_{n}^{\alpha_{n}}\right\rangle$ is a correlation function of the infinite XYZ spin chain

$$
H_{X Y Z}=\sum_{j=-\infty}^{\infty}\left(I^{1} \sigma_{j}^{1} \sigma_{j+1}^{1}+I^{2} \sigma_{j}^{2} \sigma_{j+1}^{2}+I^{3} \sigma_{j}^{3} \sigma_{j+1}^{3}\right)
$$

at zero temperature (the coefficients $I^{a}$ will be given below in (4.5)). The $h_{n}$ may be viewed as the density matrix of a finite sub-system of length $n$, regarding the rest of the spins as an environment.

From now on we fix $n$, and write $\bar{j}=2 n-j+1$. We regard $h_{n}$ as a $2^{2 n}$-dimensional vector through the identification $\operatorname{End}\left(V^{\otimes n}\right) \simeq V^{\otimes 2 n}$ given by

$$
E_{\varepsilon_{1}, \bar{\varepsilon}_{1}} \otimes \cdots \otimes E_{\varepsilon_{n}, \bar{\varepsilon}_{n}} \mapsto\left(\prod_{j=1}^{n} \bar{\varepsilon}_{j}\right) v_{\varepsilon_{1}} \otimes \cdots \otimes v_{\varepsilon_{n}} \otimes v_{-\bar{\varepsilon}_{n}} \otimes \cdots \otimes v_{-\bar{\varepsilon}_{1}},
$$

where $E_{\varepsilon, \varepsilon^{\prime}}=\left(\delta_{\varepsilon, \alpha} \delta_{\varepsilon^{\prime}, \beta}\right)_{\alpha, \beta= \pm}$.

Let us explain the constituents which enter the Ansatz.

First, we define three functions in terms of the factor $\rho(t)$ (given in (2.28) or (2.29) ) by

$$
\omega_{1}(t):=\frac{\partial}{\partial t} \log \varphi(t), \quad \omega_{2}(t):=\frac{\partial}{\partial \eta} \log \varphi(t), \quad \omega_{3}(t):=\frac{\partial}{\partial \tau} \log \varphi(t)
$$

where we have set

$$
\varphi(t):=\rho(t)^{4} \cdot \frac{\theta_{1}(2 \eta-2 t)}{\theta_{1}(2 \eta+2 t)} .
$$

They are a meromorphic solution of the system of difference equations

$$
\begin{aligned}
& \omega_{1}(t-\eta)+\omega_{1}(t)=q_{1}(t), \\
& \omega_{2}(t-\eta)+\omega_{2}(t)-\omega_{1}(t-\eta)=q_{2}(t), \\
& \omega_{3}(t-\eta)+\omega_{3}(t)=q_{3}(t),
\end{aligned}
$$

where

$$
q_{1}(t):=\frac{\partial}{\partial t} \log \psi(t), \quad q_{2}(t):=\frac{\partial}{\partial \eta} \log \psi(t), \quad q_{3}(t):=\frac{\partial}{\partial \tau} \log \psi(t)
$$

and

$$
\psi(t):=\frac{\theta_{1}(2 t)^{3} \theta_{1}(2 t-4 \eta)}{\theta_{1}(2 t-2 \eta)^{3} \theta_{1}(2 t+2 \eta)} .
$$


The next ingredient are the matrices $\widehat{X}_{a, n}^{(i, j)}(a=1,2,3,1 \leq i \neq j \leq n)$. Consider a 'transfer matrix'

$$
\begin{aligned}
& \widehat{X}_{n}\left(t_{1}, \cdots, t_{n}\right) \\
& \quad:=\frac{1}{\left[t_{1,2}\right] \prod_{p=3}^{n}\left[t_{1, p}\right]\left[t_{2, p}\right]} \operatorname{Tr}_{t_{1,2} / \eta}\left(T_{n}^{[1]}\left(\frac{t_{1}+t_{2}}{2} ; t_{1}, \cdots, t_{n}\right)\right) P_{12} \mathcal{P}_{1 \overline{1}}^{-} \mathcal{P}_{2 \overline{2}}^{-} .
\end{aligned}
$$

We used the functional $\operatorname{Tr}_{\lambda}$ introduced in the previous section, and

$$
\begin{aligned}
& T_{n}^{[1]}\left(t ; t_{1}, \ldots, t_{n}\right) \\
& \quad:=L_{\overline{2}}\left(t-t_{2}-\eta\right) \cdots L_{\bar{n}}\left(t-t_{n}-\eta\right) L_{n}\left(t-t_{n}\right) \cdots L_{2}\left(t-t_{2}\right) .
\end{aligned}
$$

Notice the presence of the permutation $P_{12}$ and the projectors $\mathcal{P}_{1 \overline{1}}^{-} \mathcal{P}_{2 \overline{2}}^{-}$in (2.34) .

For $i<j$, we define

$$
\begin{aligned}
\widehat{X}_{n}^{(i, j)}\left(t_{1}, \cdots, t_{n}\right) & =\widehat{X}_{n}^{(j, i)}\left(t_{1}, \cdots, t_{n}\right) \\
& :=\mathbb{R}_{n}^{(i, j)}\left(t_{1}, \cdots, t_{n}\right) \widehat{X}_{n}\left(t_{i}, t_{j}, t_{1}, \cdots, \widehat{t_{i}}, \cdots, \widehat{t_{j}}, \cdots, t_{n}\right) \\
& \times \mathbb{R}_{n}^{(i, j)}\left(t_{1}, \cdots, t_{n}\right)^{-1} .
\end{aligned}
$$

Here $\mathbb{R}_{n}^{(i, j)}$ stands for the product of $R$ matrices

$$
\begin{aligned}
& \mathbb{R}_{n}^{(i, j)}\left(t_{1}, \cdots, t_{n}\right) \\
& :=\check{R}_{i, i-1}\left(t_{i, i-1}\right) \cdots \check{R}_{2,1}\left(t_{i, 1}\right) \\
& \times \check{R}_{j, j-1}\left(t_{j, j-1}\right) \cdots \check{R}_{i+2, i+1}\left(t_{j, i+1}\right) \cdot \check{R}_{i+1, i}\left(t_{j, i-1}\right) \cdots \check{R}_{3,2}\left(t_{j, 1}\right) \\
& \times \check{R}_{\overline{i-1, i},}\left(t_{i-1, i}\right) \cdots \check{R}_{\overline{1} \overline{2}}\left(t_{1, i}\right) \\
& \times \check{R}_{\overline{j-1, \bar{j}}}\left(t_{j-1, j}\right) \cdots \check{R}_{\overline{i+1}, \overline{i+2}}\left(t_{i+1, j}\right) \cdot \check{R}_{\bar{i}, \overline{i+1}}\left(t_{i-1, j}\right) \cdots \check{R}_{\overline{2}, \overline{3}}\left(t_{1, j}\right) .
\end{aligned}
$$

Finally, for all $i \neq j, \widehat{X}_{a, n}^{(i j)}$ are defined by

$$
\begin{aligned}
c \widehat{X}_{1, n}^{(i, j)}\left(t_{1}, \cdots, t_{n}\right) & :=\widehat{X}_{n}^{(i, j)}\left(t_{1}, \cdots, t_{n}\right)-t_{i j} \Delta_{1}^{(i)} \widehat{X}_{n}^{(i, j)}\left(t_{1}, \cdots, t_{n}\right), \\
c \widehat{X}_{2, n}^{(i, j)}\left(t_{1}, \cdots, t_{n}\right) & =-\eta \Delta_{1}^{(i)} \widehat{X}_{n}^{(i, j)}\left(t_{1}, \cdots, t_{n}\right), \\
c \widehat{X}_{3, n}^{(i, j)}\left(t_{1}, \cdots, t_{n}\right) & :=\Delta_{\tau}^{(i)} \widehat{X}_{n}^{(i, j)}\left(t_{1}, \cdots, t_{n}\right)-\tau \Delta_{1}^{(i)} \widehat{X}_{n}^{(i, j)}\left(t_{1}, \cdots, t_{n}\right),
\end{aligned}
$$

where $c=-2 \theta_{1}^{\prime} / \theta_{1}(2 \eta)$ and

$$
\Delta_{a}^{(i)} f\left(\cdots, t_{i}, \cdots\right)=f\left(\cdots, t_{i}+a, \cdots\right)-f\left(\cdots, t_{i}, \cdots\right) .
$$

As we show in Appendix D, the $\widehat{X}_{a, n}^{(i j)}\left(t_{1}, \cdots, t_{n}\right)$ are doubly periodic in $t_{k}$ with periods $1, \tau$. The only exception is the case $a=1, k=i$ or $j$ and with respect to the shift by $\tau$, where the transformation law becomes

$$
\Delta_{\tau}^{(i)} \widehat{X}_{1, n}^{(i, j)}\left(t_{1}, \cdots, t_{n}\right)=\Delta_{-\tau}^{(j)} \widehat{X}_{1, n}^{(i, j)}\left(t_{1}, \cdots, t_{n}\right)=\widehat{X}_{3, n}^{(i, j)}\left(t_{1}, \cdots, t_{n}\right) .
$$

Conversely we have

$$
\widehat{X}_{n}^{(i j)}\left(t_{1}, \cdots, t_{n}\right)=c\left(\widehat{X}_{1, n}^{(i, j)}\left(t_{1}, \cdots, t_{n}\right)-\frac{t_{i j}}{\eta} \widehat{X}_{2, n}^{(i, j)}\left(t_{1}, \cdots, t_{n}\right)\right) .
$$


We compute the trace in the formula (2.35) by using the formulas (2.17) and (2.21). The separation of $\widehat{X}_{n}^{(i, j)}$ into two parts $\widehat{X}_{1, n}^{(i, j)}$ and $\widehat{X}_{2, n}^{(i, j)}$ comes from that of $\operatorname{Tr}_{\lambda} S_{\alpha}^{2}$ into $F_{\alpha 1}(\lambda \eta)$ and $F_{\alpha 2}(\lambda \eta)$. Note that $\widehat{X}_{1, n}^{(j, i)}=\widehat{X}_{1, n}^{(i, j)}$ and $\widehat{X}_{a, n}^{(j, i)}=-\widehat{X}_{a, n}^{(i, j)}$ for $a=2,3$.

We are now in a position to state our conjecture. Let

$$
\mathbf{s}_{n}:=\prod_{p=1}^{n} s_{p \bar{p}}
$$

be the vector corresponding to the identity by the map (2.31).

Conjecture. Correlation functions of the inhomogeneous eight-vertex model are given by the formula

$$
h_{n}\left(t_{1}, \cdots, t_{n}\right)=2^{-n} \exp \left(\sum_{i<j} \sum_{a=1}^{3} \omega_{a}\left(t_{i j}\right) \widehat{X}_{a, n}^{(i, j)}\left(t_{1}, \cdots, t_{n}\right)\right) \mathbf{s}_{n},
$$

where $\omega_{a}(t)$ and $\widehat{X}_{a, n}^{(i, j)}$ are defined respectively by (2.32) and (2.34)-(2.39).

\section{REDUCED QKZ EQUATION}

The $h_{n}$ is known to satisfy the following set of equations [16]:

$$
\begin{aligned}
& h_{n}\left(\cdots, t_{j+1}, t_{j}, \cdots\right) \\
& =\check{R}_{j, j+1}\left(t_{j, j+1}\right) \check{R}_{\overline{j+1}, \bar{j}}\left(t_{j+1, j}\right) h_{n}\left(\cdots, t_{j}, t_{j+1}, \cdots\right) \quad(1 \leq j \leq n-1), \\
& h_{n}\left(\cdots, t_{j}-\eta, \cdots\right)=A_{n}^{(j)}\left(t_{1}, \cdots, t_{n}\right) h_{n}\left(\cdots, t_{j}, \cdots\right), \\
& \mathcal{P}_{1, \overline{1}}^{-} \cdot h_{n}\left(t_{1}, \cdots, t_{n}\right)_{1, \ldots, n, \bar{n}, \ldots, \overline{1}}=\frac{1}{2} s_{1 \overline{1}} h_{n-1}\left(t_{2}, \cdots, t_{n}\right)_{2, \ldots, n, \bar{n}, \ldots, \overline{2}} .
\end{aligned}
$$

Here

$$
\begin{aligned}
& A_{n}^{(j)}\left(t_{1}, \cdots, t_{n}\right) \\
& =(-1)^{n} R_{j, j-1}\left(t_{j, j-1}-\eta\right) \cdots R_{j, 1}\left(t_{j, 1}-\eta\right) R_{\bar{j}, \overline{j+1}}\left(t_{j, j+1}-\eta\right) \cdots R_{\bar{j}, \bar{n}}\left(t_{j, n}-\eta\right) \\
& \quad \times P_{j, \bar{j}} R_{j, n}\left(t_{j, n}\right) \cdots R_{j, j+1}\left(t_{j, j+1}\right) R_{\bar{j}, \overline{1}}\left(t_{j, 1}\right) \cdots R_{\bar{j}, \overline{j-1}}\left(t_{j, j-1}\right) .
\end{aligned}
$$

In this section, assuming a conjectural identity, we explain that these relations are valid also for the Ansatz.

3.1. Properties of $\widehat{\Omega}_{n}^{(i, j)}$. Consider the expression

$$
\widehat{\Omega}_{n}^{(i, j)}\left(t_{1}, \cdots, t_{n}\right)=\sum_{a=1}^{3} \omega_{a}\left(t_{i j}\right) \widehat{X}_{a, n}^{(i, j)}\left(t_{1}, \cdots, t_{n}\right),
$$

which enters the Ansatz (2.40). In 4] for the XXZ model, the following relations are derived.

\section{Exchange relation:}

$$
\begin{aligned}
& \check{R}_{k, k+1}\left(t_{k, k+1}\right) \check{R}_{\overline{k+1}, \bar{k}}\left(t_{k+1, k}\right) \widehat{\Omega}_{n}^{(i, j)}\left(\cdots, t_{k}, t_{k+1}, \cdots\right) \\
& =\widehat{\Omega}_{n}^{\left(\pi_{k}(i), \pi_{k}(j)\right)}\left(\cdots, t_{k+1}, t_{k}, \cdots\right) \check{R}_{k, k+1}\left(t_{k, k+1}\right) \check{R}_{\overline{k+1}, \bar{k}}\left(t_{k+1, k}\right),
\end{aligned}
$$

Here $\pi_{k}$ signifies the transposition $(k, k+1)$. 


\section{Difference equations:}

$$
\begin{aligned}
& \widehat{\Omega}_{n}^{(i, j)}\left(t_{1}, \cdots, t_{k}-\eta, \cdots, t_{n}\right) \\
& \quad=A_{n}^{(k)}\left(t_{1}, \cdots, t_{n}\right) \widehat{\Omega}_{n}^{(i, j)}\left(t_{1}, \cdots, t_{n}\right) A_{n}^{(k)}\left(t_{1}, \cdots, t_{n}\right)^{-1} \quad(k \neq i, j), \\
& \widehat{\Omega}_{n}^{(i, j)}\left(t_{1}, \cdots, t_{i}-\eta, \cdots, t_{n}\right) \mathbf{s}_{n} \\
& \quad=A_{n}^{(i)}\left(t_{1}, \cdots, t_{n}\right)\left(\widehat{\Omega}_{n}^{(i, j)}\left(t_{1}, \cdots, t_{n}\right)+\widehat{Y}_{n}^{(i, j)}\left(t_{1}, \cdots, t_{n}\right)\right) \mathbf{s}_{n} .
\end{aligned}
$$

In the last line, we have set

$$
\widehat{Y}_{n}^{(i, j)}\left(t_{1}, \cdots, t_{n}\right):=\sum_{a=1}^{3} q_{a}\left(t_{i j}\right) \widehat{X}_{a, n}^{(i, j)}\left(t_{1}, \cdots, t_{n}\right),
$$

where $q_{a}(t)$ are given by (2.33).

\section{Recurrence relation:}

$$
\begin{aligned}
& \mathcal{P}_{1, \overline{1}}^{-} \widehat{\Omega}_{n}^{(i, j)}\left(t_{1}, \cdots, t_{n}\right) \\
& = \begin{cases}0 & (1=i<j \leq n), \\
\widehat{\Omega}_{n-1}^{(i-1, j-1)}\left(t_{2}, \cdots, t_{n}\right)_{2, \ldots, n, \bar{n}, \ldots, \overline{2}} \mathcal{P}_{1, \overline{1}}^{-} & (2 \leq i<j \leq n) .\end{cases}
\end{aligned}
$$

Commutativity: For distinct indices $i, j, k, l$,

$$
\widehat{\Omega}_{n}^{(i, j)}\left(t_{1}, \cdots, t_{n}\right) \widehat{\Omega}_{n}^{(k, l)}\left(t_{1}, \cdots, t_{n}\right)=\widehat{\Omega}_{n}^{(k, l)}\left(t_{1}, \cdots, t_{n}\right) \widehat{\Omega}_{n}^{(i, j)}\left(t_{1}, \cdots, t_{n}\right) .
$$

\section{Nilpotency:}

$$
\widehat{\Omega}_{n}^{(i, j)}\left(t_{1}, \cdots, t_{n}\right) \widehat{\Omega}_{n}^{(k, l)}\left(t_{1}, \cdots, t_{n}\right)=0 \quad \text { if } \quad\{i, j\} \cap\{k, l\} \neq \emptyset .
$$

The proof of these relations given in [4] are based only on the properties (2.3), (2.4)(2.7) of the $R$ matrix and (2.14), (2.15) of the $L$ operator. Hence they carry over to the elliptic case as well.

As is shown in [4, Proposition 4.1, the equations (3.5)-(3.9) guarantee the validity of the fundamental properties (3.1), (3.2), (3.3) for the Ansatz, provided one additional identity holds:

\section{Cancellation identity:}

$$
\left(\sum_{j=2}^{n} \widehat{Y}_{n}^{(1, j)}\left(t_{1}, \cdots, t_{n}\right)+\left(A_{n}^{(1)}\left(t_{1}, \cdots, t_{n}\right)^{-1}-1\right)\right) \mathbf{s}_{n}=0 .
$$

So far we have not been able to prove the cancellation identity. In the next subsection, we suggest a possible approach toward its proof.

\subsection{Cancellation identity. Set}

$$
\begin{aligned}
& Q_{n}^{(i)}\left(t_{1}, \cdots, t_{n}\right) \\
& \quad=\left(\sum_{j=2}^{n} \widehat{Y}_{n}^{(1, j)}\left(t_{1}, \cdots, t_{n}\right)+\left(A_{n}^{(1)}\left(t_{1}, \cdots, t_{n}\right)^{-1}-1\right)\right) \mathbf{s}_{n} .
\end{aligned}
$$

We regard it as a matrix via the isomorphism (2.31). 
Besides the obvious translation invariance, $Q_{n}=Q_{n}^{(1)}$ has the following properties.

$$
\begin{aligned}
& \prod_{j=2}^{n} \theta_{1}\left(2 t_{1, j}\right) \cdot Q_{n}\left(t_{1}, \cdots, t_{n}\right) \text { is entire, } \\
& Q_{n}\left(\cdots, t_{j}+\frac{1}{2}, \cdots\right)=\sigma_{j}^{1} Q_{n}\left(\cdots, t_{j}, \cdots\right) \sigma_{j}^{1}, \quad(1 \leq j \leq n), \\
& Q_{n}\left(\cdots, t_{j}+\frac{\tau}{2}, \cdots\right)=\sigma_{j}^{3} Q_{n}\left(\cdots, t_{j}, \cdots\right) \sigma_{j}^{3}, \quad(1 \leq j \leq n), \\
& \check{R}_{j, j+1}\left(t_{j, j+1}\right) Q_{n}\left(\cdots, t_{j}, t_{j+1}, \cdots\right) \\
& \quad=Q_{n}\left(\cdots, t_{j+1}, t_{j}, \cdots\right) \check{R}_{j, j+1}\left(t_{j, j+1}\right), \quad(2 \leq j \leq n-1), \\
& \left.Q_{n}\left(t_{1}, \cdots, t_{n-1}, t_{n}\right) \mathcal{P}_{n-1, n}^{-}\right|_{t_{n-1}=t_{n}+\eta}=Q_{n-2}\left(t_{1}, \cdots, t_{n-2}\right) \mathcal{P}_{n-1, n}^{-}, \\
& \operatorname{tr}_{1} Q_{n}\left(t_{1}, \cdots, t_{n}\right)=0, \\
& \operatorname{tr}_{n} Q_{n}\left(t_{1}, \cdots, t_{n}\right)=Q_{n-1}\left(t_{1}, \cdots, t_{n-1}\right) .
\end{aligned}
$$

These relations are verified in a way similar to those in [4]. The derivation of (3.13)-

(3.14) rests on the transformation laws of the $\widehat{X}_{a, n}^{(i, j)}$, which we discuss in Appendix D.

From the properties (3.12), (3.13), (3.14), $Q_{n}$ can be written as

$$
\begin{aligned}
& \prod_{j=2}^{n} \theta_{1}\left(2 t_{1 j}\right) \times Q_{n}\left(t_{1}, \cdots, t_{n}\right) \\
& \quad=\sum_{\alpha_{1}, \cdots, \alpha_{n}=0}^{3} \kappa_{\alpha_{n}, \cdots, \alpha_{1}} \prod_{j=1}^{n} \frac{\theta_{\alpha_{j}+1}\left(2 t_{1 j}\right)}{\theta_{\alpha_{j}+1}(\eta)} \sigma_{1}^{\alpha_{1}} \cdots \sigma_{n}^{\alpha_{n}},
\end{aligned}
$$

with some $\kappa_{\alpha_{n}, \cdots, \alpha_{1}} \in \mathbb{C}$. Terms with $\alpha_{1}=0$ are actually absent in the sum, in accordance with (3.17). For convenience we set $\kappa_{\alpha_{n}, \cdots, \alpha_{2}, 0}=0$. Note that (3.13), (3.14) and the translation invariance imply

$$
\kappa_{\alpha_{n}, \cdots, \alpha_{1}}=0 \quad \text { unless } \sum_{j=1}^{n} \bar{\alpha}_{j}=(0,0) .
$$

By induction, assume $Q_{m}=0$ for $m<n$. We are going to argue that $Q_{n}$ is then determined up to a multiplicative constant (see Lemma 3.1 below).

By (3.16), the induction hypothesis and (3.15), we have

$$
\left.Q_{n}\left(\cdots, t_{j}, t_{j+1}, \cdots\right) \mathcal{P}_{j, j+1}^{-}\right|_{t_{j}=t_{j+1}+\eta}=0 \quad(2 \leq j \leq n-1)
$$

By (3.18) we may also assume

$$
\kappa_{\alpha_{n}, \cdots, \alpha_{1}}=0 \quad \text { unless } \alpha_{n} \neq 0 .
$$

Quite generally, consider a matrix of the form

$$
U_{1,2}(u, v)=\sum_{\alpha, \beta=0}^{3} \kappa_{\beta \alpha} \frac{\theta_{\alpha+1}(2 u)}{\theta_{\alpha+1}(\eta)} \frac{\theta_{\beta+1}(2 v)}{\theta_{\beta+1}(\eta)} \sigma_{1}^{\alpha} \sigma_{2}^{\beta} .
$$


Then the relations

$$
\begin{aligned}
& \check{R}_{12}(u-v) U_{1,2}(u, v)=U_{1,2}(v, u) \check{R}_{12}(u-v), \\
& U_{1,2}(u+\eta, u) \mathcal{P}_{1,2}^{-}=0,
\end{aligned}
$$

are equivalent to the following relations for the coefficients $\kappa_{b a}$ :

$$
\begin{aligned}
& \kappa_{a, 0}-\kappa_{0, a}=i J_{b c}\left(\kappa_{c, b}+\kappa_{b, c}\right), \\
& \kappa_{b, a}-\kappa_{a, b}=i\left(\kappa_{c, 0}+\kappa_{0, c}\right), \\
& \sum_{\alpha=0}^{3} \kappa_{\alpha, \alpha}=0 \\
& \sum_{a=1}^{3} J_{a} \kappa_{a, a}=0 .
\end{aligned}
$$

Here $a, b, c$ are cyclic permutations of $1,2,3$, and $J_{a}, J_{b c}$ are as in (2.10), (2.11). The above relations have the same form as those derived from the quadratic relations (2.8),(2.9) and from the Casimir elements (2.13), respectively. Consider the quotient $\overline{\mathcal{A}}$ of the Sklyanin algebra modulo the relations that the Casimir elements are zero. This is a graded algebra,

$$
\overline{\mathcal{A}}=\bigoplus_{n=0}^{\infty} \overline{\mathcal{A}}_{n} .
$$

From the above considerations one easily concludes that there exist three linear functionals $\kappa_{a}(a=1,2,3)$ on $\overline{\mathcal{A}}_{n-1}$ such that

$$
\kappa_{\alpha_{n}, \cdots, \alpha_{2}, a}=\kappa_{a}\left(S_{\alpha_{n}} \cdots S_{\alpha_{2}}\right)
$$

which satisfy the additional condition

$$
\kappa_{a}\left(S_{0} A\right)=0 .
$$

In the Sklyanin algebra with generic parameter $\eta$, any monomial $S_{\alpha_{2}} \cdots S_{\alpha_{n}}$ can be reduced to a linear combination of ordered monomials $S_{0}^{\nu_{0}} S_{3}^{\nu_{3}} S_{1}^{\nu_{2}} S_{2}^{\nu_{1}}$ with $\nu_{1}, \nu_{2} \in$ $\{0,1\}$, by using the quadratic relations and Casimir elements (PBW basis) [10, 11]. Together with (3.20) this means that each functional $\kappa_{a}$ is defined by one constant, that is,

$$
\begin{array}{lr}
\kappa_{1}\left(S_{3}^{n-2} S_{1}\right), \kappa_{2}\left(S_{3}^{n-2} S_{2}\right), \kappa_{3}\left(S_{3}^{n-1}\right) & \text { for } n \text { even, } \\
\kappa_{1}\left(S_{3}^{n-2} S_{2}\right), \kappa_{2}\left(S_{3}^{n-2} S_{1}\right), \kappa_{3}\left(S_{3}^{n-3} S_{1} S_{2}\right) & \text { for } n \text { odd. }
\end{array}
$$

There remain three coefficients. In order to finish the proof of the Cancellation Identity, it remains to show that these coefficients vanish.

In addition to (3.15), we have also the relation

$$
\check{R}_{12}\left(\lambda_{12}\right) Q_{n}\left(t_{1}, t_{2}, \cdots\right) \check{R}_{12}\left(\lambda_{12}\right)^{-1}=Q_{n}^{(2)}\left(t_{2}, t_{1}, \cdots\right) .
$$

The poles of the $R$ matrix in the left hand side are not the poles of $Q_{n}^{(2)}$. This entails the relation

$$
\mathcal{P}_{12}^{-} Q_{n}\left(t_{1}, t_{2}, \cdots\right) r_{12}(1)=0
$$


which can be rewritten in terms of functionals $\kappa_{a}$ as follows:

$$
2 \kappa_{a}\left(A S_{0}\right)-i\left(1+J_{b c}\right) \kappa_{c}\left(A S_{b}\right)+i\left(1-J_{b c}\right) \kappa_{b}\left(A S_{c}\right)=0 \quad \forall A \in \overline{\mathcal{A}}_{n-2} .
$$

These equations can be viewed as a system of linear equations for three constants (3.25). Certainly, these equations are not explicit since for every $A$ we have to perform the procedure of reducing to PBW form. This huge system of homogeneous linear equations does not allow us to prove that the constants in question vanish; rather they reduce them to one constant. Let us explain this point. First, it is clear that the equations (3.26) correspond to the following relation in $\overline{\mathcal{A}}_{n}$

$$
2 S_{0} S_{a}-i\left(1+J_{b c}\right) S_{b} S_{c}+i\left(1-J_{b c}\right) S_{c} S_{b}=0,
$$

obtained by solving (2.8), (2.9) for $S_{0} S_{a}$. So, it is easy to see that all our equations including (3.26) are satisfied by the following construction. Consider a linear functional $\kappa$ on $\overline{\mathcal{A}}_{n}$ such that $\kappa\left(S_{\alpha_{1}} \cdots S_{\alpha_{n}}\right)=0$ unless $\sum_{j=1}^{n} \bar{\alpha}_{j}=(0,0)$, and

$$
\kappa\left(A S_{0}\right)=0 .
$$

Then all the requirements are satisfied by

$$
\kappa_{a}(A)=\kappa\left(A S_{a}\right) .
$$

On the other hand the number of solutions to the system of linear equations (3.26) for three constants cannot be bigger for arbitrary $\eta$ than it is for $\eta=0$. In the latter case the algebra is commutative (see Appendix A notably (A.4)), and the equations (3.26) become

$$
\kappa_{a}^{\mathrm{cl}}\left(S_{b} A\right)=\kappa_{b}^{\mathrm{cl}}\left(S_{a} A\right)
$$

with additional condition $\kappa_{a}^{\mathrm{cl}}\left(S_{0} A\right)=0$. It is easy to see that this gives a system of three equations for three constants whose rank equals 2 .

Thus we come to the conclusion:

Lemma 3.1. Under the induction hypothesis, we have

$$
\kappa_{\alpha_{n}, \cdots, \alpha_{1}}=\kappa\left(S_{\alpha_{n}} \cdots S_{\alpha_{1}}\right)
$$

where $\kappa$ is a linear functional on $\overline{\mathcal{A}}_{n}$ satisfying

$$
\begin{aligned}
& \kappa\left(S_{\alpha_{n}} \cdots S_{\alpha_{1}}\right)=0 \quad \text { unless } \sum_{j=1}^{n} \bar{\alpha}_{j}=(0,0) \\
& \kappa\left(A S_{0}\right)=\kappa\left(S_{0} A\right)=0
\end{aligned}
$$

and as such is defined by one constant:

$$
\begin{array}{ll}
\kappa\left(S_{3}^{n}\right) & \text { for } n \text { even, } \\
\kappa\left(S_{3}^{n-2} S_{1} S_{2}\right) & \text { for } n \text { odd. }
\end{array}
$$

Unfortunately, we were not able to show that this remaining constant equals zero. The problem is still open. 


\section{EXAMPLES}

In this section we write down the Ansatz in the simplest cases $n=2,3$. We also consider the trigonometric limit.

4.1. The case $n=2$. In the case $n=2, \Omega_{2}^{(1,2)}\left(t_{1}, t_{2}\right)$ can be readily found from (2.21). The function $h_{2}\left(t_{1}, t_{2}\right)$ is given as follows:

$$
h_{2}\left(t_{1}, t_{2}\right)=\frac{1}{4}-\frac{1}{4\left[t_{12}\right]} \sum_{a=1}^{3} H_{a+1}\left(2 t_{12}\right) \sigma^{a} \otimes \sigma^{a},
$$

where

$$
H_{a+1}(2 t):=\frac{\varepsilon_{a} \theta_{a+1}^{2} \theta_{a+1}(2 \eta) \theta_{a+1}(2 t)}{4\left(\theta_{1}^{\prime}\right)^{2} \theta_{1}(2 \eta)^{2}}\left(\frac{\theta_{a+1}^{\prime}(2 t)}{\theta_{a+1}(2 t)} \omega_{1}(t)+\frac{\theta_{a+1}^{\prime}(2 \eta)}{\theta_{a+1}(2 \eta)} \omega_{2}(t)-4 \pi i \omega_{3}(t)\right) .
$$

This gives the formula for the nearest neighbor correlators of the inhomogeneous chain:

$$
\left\langle\sigma_{1}^{a} \sigma_{2}^{a}\right\rangle=-\frac{\theta_{1}(2 \eta)}{\theta_{1}(2 t)} H_{a+1}(2 t)
$$

where $a=1,2,3$ and $t=t_{12}$. Noting that $H_{a+1}(2 t)$ is odd in $t$, we obtain $\left\langle\sigma_{1}^{a} \sigma_{2}^{a}\right\rangle=$ $-\theta_{1}(2 \eta) H_{a+1}^{\prime}(0) / \theta_{1}^{\prime}$ in the homogeneous limit $t \rightarrow 0$, or more explicitly we have

$$
\begin{aligned}
& \left\langle\sigma_{1}^{a} \sigma_{2}^{a}\right\rangle=-\frac{\varepsilon_{a} \theta_{a+1}^{2}}{8 \theta_{1}^{\prime 3} \theta_{1}(2 \eta)} \\
& \quad \times\left(2 \theta_{a+1}^{\prime \prime}(0) \theta_{a+1}(2 \eta)+\theta_{a+1} \theta_{a+1}^{\prime}(2 \eta) \frac{\partial}{\partial \eta}-4 \pi i \theta_{a+1} \theta_{a+1}(2 \eta) \frac{\partial}{\partial \tau}\right) \omega_{1}(0) .
\end{aligned}
$$

Let us check the formula (4.2) against known results. As is well known, the XYZ Hamiltonian is obtained by differentiating the transfer matrix of the eight-vertex model

$$
T_{L}(t)=\operatorname{tr}\left(R_{0 L}(t) \cdots R_{01}(t)\right)
$$

as

$$
T_{L}(0)^{-1} T_{L}^{\prime}(0)=\sum_{j=1}^{L}\left(\sum_{a=1}^{3} v_{a}^{\prime}(0) \sigma_{j}^{a} \sigma_{j+1}^{a}\right)+L v_{0}^{\prime}(0),
$$

where $L$ is the length of the chain, and we have set $\check{R}(t)=\sum_{\alpha=0}^{3} v_{\alpha}(t) \sigma^{\alpha} \otimes \sigma^{\alpha}$. As it was mentioned already, the $R$ matrix (2.11) is so normailized that in the thermodynamic limit $L \rightarrow \infty$ the free energy per site of the eight-vertex model is 0 . Therefore, taking the ground state average of (4.3), we obtain

$$
\sum_{a=1}^{3} I^{a}\left\langle\sigma_{1}^{a} \sigma_{2}^{a}\right\rangle=-I^{0}
$$


with $I^{\alpha}=v_{\alpha}^{\prime}(0) \theta_{1}(2 \eta) / \theta_{1}^{\prime}$. Explicitly we have

$$
\begin{aligned}
I^{a} & =\frac{\theta_{a+1}(2 \eta)}{\theta_{a+1}} \quad(a=1,2,3), \\
I^{0} & =\frac{\theta_{1}(2 \eta)}{\theta_{1}^{\prime}} \frac{1}{4} \omega_{1}(0) .
\end{aligned}
$$

The average over the normalized ground state has the property $\delta\left\langle H_{X Y Z}\right\rangle=\left\langle\delta H_{X Y Z}\right\rangle$, where $\delta$ stands for the variation of the coefficients $I^{a}$. Hence we have in addition

$$
\begin{aligned}
& \sum_{a=1}^{3} \frac{\partial I^{a}}{\partial \eta}\left\langle\sigma_{1}^{a} \sigma_{2}^{a}\right\rangle=-\frac{\partial I^{0}}{\partial \eta} \\
& \sum_{a=1}^{3} \frac{\partial I^{a}}{\partial \tau}\left\langle\sigma_{1}^{a} \sigma_{2}^{a}\right\rangle=-\frac{\partial I^{0}}{\partial \tau}
\end{aligned}
$$

The nearest neighbor correlators $\left\langle\sigma_{1}^{a} \sigma_{2}^{a}\right\rangle$ are completely determined by the linear equations (4.4), (4.6), (4.7). Using Riemann's identity and the heat equation $4 \pi i \partial \theta_{\alpha}(t \mid \tau) / \partial \tau=\partial^{2} \theta_{\alpha}(t \mid \tau) / \partial t^{2}$, one can verify that our formula (4.2) indeed gives the unique solution.

4.2. The case $n=3$. Let us proceed to the next case $n=3$. Written in full, $h_{3}\left(t_{1}, t_{2}, t_{3}\right)$ reads

$$
h_{3}\left(t_{1}, t_{2}, t_{3}\right)=\frac{1}{8}-\frac{1}{16} \frac{1}{\left[t_{12}\right]\left[t_{13}\right]\left[t_{23}\right]} \sum_{\substack{\alpha, \beta, \gamma) \neq(0,0,0) \\ \bar{\alpha}+\bar{\beta}+\bar{\gamma}=\overline{0}}} \sigma^{\alpha} \otimes \sigma^{\beta} \otimes \sigma^{\gamma} \sum_{1 \leq j<k \leq 3} I_{\alpha, \beta, \gamma}^{(j, k)}\left(t_{1}, t_{2}, t_{3}\right) .
$$

The coefficients $I_{\alpha, \beta, \gamma}^{(j, k)}$ are given as follows:

$$
\begin{aligned}
I_{0,1,1}^{(1,2)} & =0 \\
I_{1,0,1}^{(1,2)} & =\frac{\theta_{2}}{\theta_{2}(2 \eta)}\left\{\frac{\theta_{4}\left(2 t_{13}\right) \theta_{3}\left(2 t_{23}\right)}{\theta_{4}(2 \eta) \theta_{3}} H_{3}\left(2 t_{12}\right)+\frac{\theta_{3}\left(2 t_{13}\right) \theta_{4}\left(2 t_{23}\right)}{\theta_{3}(2 \eta) \theta_{4}} H_{4}\left(2 t_{12}\right)\right\} \\
I_{1,1,0}^{(1,2)} & =2\left[t_{13}\right]\left[t_{23}\right] H_{2}\left(2 t_{12}\right), \\
I_{1,2,3}^{(1,2)} & =(-i)\left\{\left[t_{13}\right] \frac{\theta_{4}\left(2 t_{23}\right)}{\theta_{4}(2 \eta)} H_{2}\left(2 t_{12}\right)-\frac{\theta_{2} \theta_{3}(2 \eta)}{\theta_{2}(2 \eta) \theta_{3}} \frac{\theta_{4}\left(2 t_{13}\right)}{\theta_{4}(2 \eta)}\left[t_{23}\right] H_{3}\left(2 t_{12}\right)\right\} \\
I_{1,3,2}^{(1,2)} & =(-i)\left\{\frac{\theta_{2} \theta_{4}(2 \eta)}{\theta_{2}(2 \eta) \theta_{4}} \frac{\theta_{3}\left(2 t_{13}\right)}{\theta_{3}(2 \eta)}\left[t_{23}\right] H_{4}\left(2 t_{12}\right)-\left[t_{13}\right] \frac{\theta_{3}\left(2 t_{23}\right)}{\theta_{3}(2 \eta)} H_{2}\left(2 t_{12}\right)\right\}
\end{aligned}
$$




$$
\begin{aligned}
I_{0,1,1}^{(1,3)}= & I_{1,1,0}^{(1,3)}=0, \\
I_{1,0,1}^{(1,3)}= & \frac{\theta_{3}^{2}(2 \eta) \theta_{4}^{2}+\theta_{4}^{2}(2 \eta) \theta_{3}^{2}}{\theta_{3} \theta_{4} \theta_{3}(2 \eta) \theta_{4}(2 \eta)}\left[t_{12}\right]\left[t_{23}\right] H_{2}\left(2 t_{13}\right) \\
& -\frac{\theta_{2}}{\theta_{2}(2 \eta)}\left\{\frac{\theta_{4}\left(2 t_{12}\right) \theta_{4}\left(2 t_{23}\right)}{\theta_{4}(2 \eta) \theta_{4}} H_{3}\left(2 t_{13}\right)+\frac{\theta_{3}\left(2 t_{12}\right) \theta_{3}\left(2 t_{23}\right)}{\theta_{3}(2 \eta) \theta_{3}} H_{4}\left(2 t_{13}\right)\right\}, \\
I_{1,2,3}^{(1,3)}= & (-i)\left\{\frac{\theta_{2}}{\theta_{3}} \frac{\theta_{3}\left(2 t_{12}\right)}{\theta_{2}(2 \eta)}\left[t_{23}\right] H_{4}\left(2 t_{13}\right)-\left[t_{12}\right] \frac{\theta_{4}}{\theta_{3}} \frac{\theta_{3}\left(2 t_{23}\right)}{\theta_{4}(2 \eta)} H_{2}\left(2 t_{13}\right)\right\} \\
I_{1,3,2}^{(1,3)}= & (-i)\left\{\left[t_{12}\right] \frac{\theta_{3}}{\theta_{4}} \frac{\theta_{4}\left(2 t_{23}\right)}{\theta_{3}(2 \eta)} H_{2}\left(2 t_{13}\right)-\frac{\theta_{2}}{\theta_{4}} \frac{\theta_{4}\left(2 t_{12}\right)}{\theta_{2}(2 \eta)}\left[t_{23}\right] H_{3}\left(2 t_{13}\right)\right\} \\
I_{1,1,0}^{(2,3)}= & \left.0, \quad \theta_{2}^{(2,3)=}=\frac{\theta_{3}\left(2 t_{12}\right) \theta_{4}\left(2 t_{13}\right)}{\theta_{3} \theta_{4}(2 \eta)} H_{3}\left(2 t_{23}\right)+\frac{\theta_{4}\left(2 t_{12}\right) \theta_{3}\left(2 t_{13}\right)}{\theta_{3}(2 \eta) \theta_{4}} H_{4}\left(2 t_{23}\right)\right\} \\
I_{0,1,1}^{(2,3)}= & 2\left[t_{12}\right]\left[t_{13}\right] H_{2}\left(2 t_{23}\right), \\
I_{1,2,3}^{(2,3)}= & (-i)\left\{\frac{\theta_{3}(2 \eta) \theta_{4} \theta_{2}\left(2 t_{13}\right)}{\theta_{3} \theta_{4}(2 \eta)}\left[t_{12}\right] H_{3}\left(2 t_{23}\right)-\left[t_{13}\right] \frac{\theta_{2}\left(2 t_{12}\right)}{\theta_{2}(2 \eta)} H_{4}\left(2 t_{23}\right)\right\} \\
I_{1,3,2}^{(2,3)}= & (-i)\left\{\left[t_{13}\right] \frac{\theta_{2}\left(2 t_{12}\right)}{\theta_{2}(2 \eta)} H_{3}\left(2 t_{2,3}\right)-\frac{\theta_{3} \theta_{4}(2 \eta)}{\theta_{3}(2 \eta) \theta_{4}} \frac{\theta_{2}\left(2 t_{13}\right)}{\theta_{2}(2 \eta)}\left[t_{12}\right] H_{4}\left(2 t_{23}\right)\right\} .
\end{aligned}
$$

The rest are given by the cyclic change $1 \rightarrow 2 \rightarrow 3 \rightarrow 1$ of the indices $\alpha, \beta, \gamma$ in $I_{\alpha, \beta, \gamma}^{(j, k)}$ with the change $2 \rightarrow 3 \rightarrow 4 \rightarrow 2$ of the indices in $\theta_{a}$ and $H_{a}$. The correlators of the inhomogeneous chain are

$$
\left\langle\sigma_{1}^{\alpha} \sigma_{2}^{\beta} \sigma_{3}^{\gamma}\right\rangle=-\frac{1}{2\left[t_{12}\right]\left[t_{13}\right]\left[t_{23}\right]} \sum_{1 \leq j<k \leq 3} I_{\alpha, \beta, \gamma}^{(j, k)}\left(t_{1}, t_{2}, t_{3}\right) .
$$

With the abbreviation $H_{a}^{\prime}=H_{a}^{\prime}(0)$ and $H_{a}^{\prime \prime \prime}=H_{a}^{\prime \prime \prime}(0)$, we obtain a new formula for the next nearest neighbor correlators for the homogeneous chain

$$
\begin{aligned}
&\left\langle\sigma_{1}^{a} \sigma_{3}^{a}\right\rangle=-\frac{1}{4} \frac{\theta_{1}(2 \eta)}{\theta_{1}^{\prime}}\left\{2 \frac{\theta_{b+1}^{2}(2 \eta) \theta_{c+1}^{2}+\theta_{c+1}^{2}(2 \eta) \theta_{b+1}^{2}}{\theta_{b+1} \theta_{c+1} \theta_{b+1}(2 \eta) \theta_{c+1}(2 \eta)} H_{a+1}^{\prime}\right. \\
&+\left(\frac{\theta_{1}(2 \eta)}{\theta_{1}^{\prime}}\right)^{2} \frac{\theta_{a+1}}{\theta_{a+1}(2 \eta)}\left\{\frac{\theta_{c+1}}{\theta_{c+1}(2 \eta)}\left(\frac{\theta_{b+1}^{\prime \prime}}{\theta_{b+1}} H_{b+1}^{\prime}+\frac{2 \theta_{c+1}^{\prime \prime}}{\theta_{c+1}} H_{b+1}^{\prime}-H_{b+1}^{\prime \prime \prime}\right)\right. \\
&\left.\left.\quad+\frac{\theta_{b+1}}{\theta_{b+1}(2 \eta)}\left(\frac{\theta_{c+1}^{\prime \prime}}{\theta_{c+1}} H_{c+1}^{\prime}+\frac{2 \theta_{b+1}^{\prime \prime}}{\theta_{b+1}} H_{c+1}^{\prime}-H_{c+1}^{\prime \prime \prime}\right)\right\}\right\} .
\end{aligned}
$$

We have in addition

$$
\left\langle\sigma_{1}^{a} \sigma_{2}^{b} \sigma_{3}^{c}\right\rangle=0, \quad\left\langle\sigma_{1}^{c} \sigma_{2}^{b} \sigma_{3}^{a}\right\rangle=0 .
$$

In both formulas, $(a, b, c)=(1,2,3),(2,3,1),(3,1,2)$.

M. Lashkevich communicated to us a program for numerically calculating correlation functions from the integral formula of [25, 26]. For $n=2$ and $n=3$, we found agreement between their results and ours to within the precision $10^{-4}$. 
4.3. Trigonometric limit. Finally we briefly touch upon the trigonometric limit, and discuss how various quantities which appear in (4.1) are related to the trigonometric counterpart.

First we consider the limit to the massive regime. For this purpose, it is convenient to rewrite the $R$ matrix in terms of the parameters $t^{\prime}, \eta^{\prime}, \tau^{\prime}$ in (2.30) as

$$
R(t)=\frac{\rho^{\prime}\left(t^{\prime}\right)}{\left[t^{\prime}+\eta^{\prime}\right]^{\prime}}(U \otimes U) r^{\prime}\left(t^{\prime}\right)(U \otimes U)^{-1},
$$

where $\left[t^{\prime}\right]^{\prime}=\theta_{1}\left(2 t^{\prime} \mid \tau^{\prime}\right) / \theta_{1}\left(2 \eta^{\prime} \mid \tau^{\prime}\right), r^{\prime}\left(t^{\prime}\right)$ is obtained from (2.2) by replacing $t, \eta, \tau$ by $t^{\prime}, \eta^{\prime}, \tau^{\prime}$, and $U=\left(\begin{array}{cc}1 & 1 \\ 1 & -1\end{array}\right)$. In the limit $\tau^{\prime} \rightarrow+i \infty$ while keeping $\lambda=t^{\prime} / \eta^{\prime}$ and $\nu=2 \eta^{\prime}$ fixed, the $R$ matrix tends to

$$
R_{\mathrm{XXZ}}(\lambda)=\rho_{\mathrm{XXZ}}(\lambda) \frac{r_{\mathrm{XXZ}}(\lambda)}{[\lambda+1]_{X X Z}}
$$

In the above, $[\lambda]_{X X Z}=\sin \pi \nu \lambda / \sin \pi \nu$, and

$$
\begin{aligned}
& r_{\mathrm{XXZ}}(\lambda)=\frac{1}{2}\left(\frac{\sin (\lambda+1 / 2) \pi \nu}{\sin \pi \nu / 2} \sigma^{0} \otimes \sigma^{0}\right. \\
& \left.+\sigma^{1} \otimes \sigma^{1}+\sigma^{2} \otimes \sigma^{2}+\frac{\cos (\lambda+1 / 2) \pi \nu}{\cos \pi \nu / 2} \sigma^{3} \otimes \sigma^{3}\right) \\
& \rho_{\mathrm{XXZ}}(\lambda)=-\zeta \frac{\left(q^{2} \zeta^{2}\right)_{\infty}\left(\zeta^{-2}\right)_{\infty}}{\left(q^{2} \zeta^{-2}\right)_{\infty}\left(\zeta^{2}\right)_{\infty}}
\end{aligned}
$$

where $\zeta=e^{\pi i \nu \lambda}, q=e^{\pi i \nu},(x)_{\infty}=\prod_{j=0}^{\infty}\left(1-q^{4 j} x\right)$. It is easy to see that

$$
\omega_{1}(t) \rightarrow \frac{8 \pi}{\sin \pi \nu} \omega(\lambda), \quad \omega_{2}(t) \rightarrow \frac{8 \pi}{\sin \pi \nu} \tilde{\omega}(\lambda), \quad \omega_{3}(t) \rightarrow 0
$$

where the functions $\omega(\lambda), \tilde{\omega}(\lambda)$ are given by [4], eqs.(13.2)-(13.5) for the massive regime. Hence the limit of (4.1) becomes

$$
\begin{aligned}
& \lim _{\tau^{\prime} \rightarrow i \infty}\left\langle\sigma_{1}^{3} \sigma_{2}^{3}\right\rangle=-4\left(\frac{q+q^{-1}}{\left(q-q^{-1}\right)^{2}} \omega(\lambda)+\frac{\zeta+\zeta^{-1}}{\left(q-q^{-1}\right)\left(\zeta-\zeta^{-1}\right)} \tilde{\omega}(\lambda)\right), \\
& \lim _{\tau^{\prime} \rightarrow i \infty}\left\langle\sigma_{1}^{1} \sigma_{2}^{1}\right\rangle=\lim _{\tau^{\prime} \rightarrow i \infty}\left\langle\sigma_{1}^{2} \sigma_{2}^{2}\right\rangle=2\left(\frac{\zeta+\zeta^{-1}}{\left(q-q^{-1}\right)^{2}} \omega(\lambda)+\frac{q+q^{-1}}{\left(q-q^{-1}\right)\left(\zeta-\zeta^{-1}\right)} \tilde{\omega}(\lambda)\right),
\end{aligned}
$$

which reproduces the formulas in the massive regime (see 4], Example in Section 3).

Second let us consider the limit to the massless regime. We set

$$
\tau=-\frac{1}{\pi i} r, \quad \eta=-\frac{\nu}{2 \pi i} r, \quad t=-\frac{\nu \lambda}{2 \pi i} r
$$

for a constant $\nu(0<\nu<1)$ and take the limit $r \downarrow 0$ with $\nu$ and $\lambda$ fixed.

The limit of the $R$ matrix is given by the same formula (4.8)-(4.9), with $\rho_{\mathrm{Xxz}}(\lambda)$ being replaced by

$$
\rho_{\mathrm{XXZ}}(\lambda)=-\frac{S_{2}(-\lambda) S_{2}(1+\lambda)}{S_{2}(\lambda) S_{2}(1-\lambda)} .
$$


Here $S_{2}(x)=S_{2}(x \mid 2,1 / \nu)$ signifies the double sine function. In the limit we have

$$
r \omega_{1}(t) \rightarrow-\frac{8 \pi^{2} i}{\sin \pi \nu} \omega(\lambda), \quad r \omega_{2}(t) \rightarrow-\frac{8 \pi^{2} i}{\sin \pi \nu} \tilde{\omega}(\lambda),
$$

where now $\omega(\lambda)$ and $\tilde{\omega}(\lambda)$ stand for the functions given by [4], eqs.(13.2)-(13.5) for the massless regime. Moreover we have

$$
r e^{\frac{\pi^{2}}{r}}\left(t \frac{\partial}{\partial t}+\eta \frac{\partial}{\partial \eta}+\tau \frac{\partial}{\partial \tau}\right) \log \varphi \rightarrow 0 .
$$

From the formulas above, we see that in the massless limit the function $h_{2}\left(t_{1}, t_{2}\right)$ tends to the solution $h_{2}\left(\lambda_{1}, \lambda_{2}\right)$ of the reduced qKZ equation given in [4].

\section{Appendix A. Existence OF $\operatorname{Tr}_{\lambda}$}

For every finite-dimensional representation of the Sklyanin algebra $\mathcal{A}$, we can define the trace, which is a functional on $\mathcal{A}$ whose main property is cyclicity. In order to formulate our Anstaz for correlation functions, we need an analytic continuation of this functional with respect to the dimension. We denote this analytic continuation by $\operatorname{Tr}_{\lambda} A$, where $A \in \mathcal{A}$ and $\lambda=k+1$ for $\pi^{(k)}(A)$. In Section 2.3. we presented the formulas for $\operatorname{Tr}_{\lambda} S_{\alpha}, \operatorname{Tr}_{\lambda} S_{\alpha}^{2}(\alpha=0,1,2,3)$. In this appendix, we discuss the general case. In fact, we prove that for generic parameters $J_{1}, J_{2}, J_{3}$, the definition of $\operatorname{Tr}_{\lambda} A$ for general $A \in \mathcal{A}$ can be reduced to these known cases.

Consider the polynomial ring $\mathbf{F}=\mathbf{K}\left[K_{0}, K_{2}\right]$ with $\mathbf{K}=\mathbb{C}\left(J_{1}, J_{2}, J_{3}\right)$. Here, we consider $K_{0}, K_{2}, J_{1}, J_{2}, J_{3}$ as variables, whereas they are parameterized by $\tau, \eta$ and $\lambda$ in Section 2 and Appendix B. We use the parameterization in order to define finite dimensional representations. The discussion in this appendix is mainly concerned with the algebraic relations in the Sklyanin algebra only.

We denote by $\mathbf{A}$ the Sklyanin algebra defined over the field $\mathbf{K}$. It is a graded vector space,

$$
\mathbf{A}=\oplus_{n=0}^{\infty} \mathbf{A}_{n}, \quad \operatorname{dim} \mathbf{A}_{n}<\infty .
$$

Multiplication by the central elements (2.13) endows A with an F-algebra structure. Suppose we try to define some $\mathbf{F}$-linear functional $\operatorname{Tr}$ on $\mathbf{A}$ which satisfies cyclicity $\operatorname{Tr}(A B)=\operatorname{Tr}(B A)$. Then the question is, for how many independent elements of $\mathbf{A}$ this functional should be defined. In other words, describe the $\mathbf{F}$-module

$$
\mathbf{H}=\mathbf{A} / \mathbf{A}^{\prime}
$$

where

$$
\mathbf{A}^{\prime}=\sum_{\alpha=0}^{3}\left[S_{\alpha}, \mathbf{A}\right]
$$

Note that $\mathbf{H}=\oplus_{n=0}^{\infty} \mathbf{H}_{n}$ where $\mathbf{H}_{n}=\mathbf{A}_{n} / \mathbf{A}_{n}^{\prime}, \mathbf{A}_{n}^{\prime}=\sum_{\alpha=0}^{3}\left[S_{\alpha}, \mathbf{A}_{n-1}\right]$.

We prove

Theorem A.1. The $\mathbf{F}$-module $\mathbf{H}$ is a rank 7 free module generated by the monomials

$$
\left(m_{i}\right)_{1 \leq i \leq 7}=\left(1, S_{0}, S_{1}, S_{2}, S_{3}, S_{0}^{2}, S_{3}^{2}\right) .
$$


The F-linear independence of these elements follows from (2.20)-2.23). Indeed, suppose there is a relation $\sum_{i=1}^{7} c_{i} m_{i}=0$ with $c_{i} \in \mathbf{F}$. The sum over elements of even degree and of odd degree must vanish separately. Specialize $J_{i}$ to the value (2.11) with $\eta \notin \mathbb{Q}+\mathbb{Q} \tau, \operatorname{Im} \eta>0$, and take the trace of both sides on the representation $\mathcal{V}^{(k)}$ for $k \in \mathbb{Z}_{\geq 0}$. By Lemma C.2, it follows that $c_{i}=0$ except for $i=3,4,5$. To see that the latter vanish, it is enough to apply the automorphisms $\iota^{1}, \iota^{3}$ (see (D.1), (D.2) and two lines above) and take the trace.

Let us prove the spanning property.

Consider the tensor algebra $\mathbf{T}$ over the field $\mathbf{K}$ generated by four independent variables $S_{0}, S_{1}, S_{2}$ and $S_{3}$. Set $\mathbf{R}=\mathbf{T}\left[K_{0}, K_{2}\right]$. It is a graded algebra: $\mathbf{R}=\oplus_{n=0}^{\infty} \mathbf{R}_{n}$, where we have $\operatorname{dim}_{\mathbf{K}} \mathbf{R}_{n}<\infty$. We have the isomorphism of $\mathbf{K}$-vector spaces

$$
\begin{array}{r}
\mathbf{H}_{n} \simeq \mathbf{R}_{n} /\left(\sum \mathbf{R}_{n-2}\left(\left[S_{0}, S_{a}\right]-i J_{b, c}\left(S_{b} S_{c}+S_{c} S_{b}\right)\right)\right. \\
+\sum \mathbf{R}_{n-2}\left(\left[S_{b}, S_{c}\right]-i\left(S_{0} S_{a}+S_{a} S_{0}\right)\right)+\sum\left[S_{a}, \mathbf{R}_{n-1}\right] \\
\left.+\mathbf{R}_{n-2}\left(\sum_{\alpha=0}^{3} S_{\alpha}^{2}-K_{0}\right)+\mathbf{R}_{n-2}\left(\sum_{a=1}^{3} J_{a} S_{a}^{2}-K_{2}\right)\right) .
\end{array}
$$

The $\mathbf{K}$-vector space $\mathbf{R}_{n}$ is spanned by the monomials of the form $K_{0}^{m_{0}} K_{2}^{m_{2}} S_{\alpha_{1}} \cdots S_{\alpha_{l}}$ where $2 m_{0}+2 m_{2}+l=n$. The relations which define $\mathbf{H}_{n}$ in (A.2) are linear relations for these monomials. For each $n$ the coefficients of these linear relations form a matrix $\mathcal{M}_{n}$ with entries in $\mathbf{K}$.

The spanning property is clear for $n=0,1$. Suppose that $n \geq 2$. Divide the set of monomials of degree $n$ into two groups: the first group is the monomials such that the part $S_{\alpha_{1}} \cdots S_{\alpha_{l}}$ is equal to one of $m_{i}(1 \leq i \leq 7)$ and the second group is the rest. The matrix $\mathcal{M}_{n}$ is divided into two blocks $\overline{\mathcal{M}}_{n}=\left(\mathcal{M}_{n}^{\prime}, \mathcal{M}_{n}^{\prime \prime}\right)$ where $\mathcal{M}_{n}^{\prime}$ (resp., $\mathcal{M}_{n}^{\prime \prime}$ ) corresponds to the first (resp., the second) group of monomials. It suffices to show that the rank of $\mathcal{M}_{n}^{\prime \prime}$ is equal to the cardinality of the second group.

The proof of this statement exploits the classical limit:

$$
J_{a}=1-\varepsilon^{2} j_{a}, \quad \varepsilon \rightarrow 0 .
$$

We introduce new variables $s_{\alpha}(\alpha=0,1,2,3)$ and $k_{0}, k_{1}$ by

$$
\begin{aligned}
& S_{0}=\varepsilon s_{0}, \quad S_{a}=s_{a} \quad(a=1,2,3), \\
& K_{0}=k_{0}, \quad K_{2}=k_{0}-\varepsilon^{2} k_{1} .
\end{aligned}
$$

In Appendix $\mathbb{B}$, the classical limit is taken as $\eta \rightarrow 0$ instead of $\varepsilon \rightarrow 0$. In this appendix, we avoid the parametrization by $\tau, \eta$ and $\lambda$ in order to simplify the argument.

In the limit $\varepsilon \rightarrow 0$, we have

$$
\left[s_{\alpha}, s_{\beta}\right]=i \varepsilon\left\{s_{\alpha}, s_{\beta}\right\}+O\left(\varepsilon^{2}\right),
$$

where the Poisson bracket is defined by

$$
\begin{aligned}
& \left\{s_{0}, s_{a}\right\}=2 j_{b, c} s_{b} s_{c} \\
& \left\{s_{b}, s_{c}\right\}=2 s_{0} s_{a} .
\end{aligned}
$$


Here $j_{a, b}=-j_{b, a}=j_{a}-j_{b}$ and $(a, b, c)$ runs over cyclic permutations of $(1,2,3)$. In the classical limit, the variables $s_{a}$ become commutative. Let $\mathbf{K}^{\mathrm{cl}}=\mathbb{C}\left(j_{1}, j_{2}, j_{3}\right)$ denote the field of rational functions in $j_{a}$, and let $\mathbf{F}^{\mathrm{cl}}=\mathbf{K}^{\mathrm{cl}}\left[k_{0}, k_{1}\right], \mathbf{A}^{\mathrm{cl}}=\mathbf{K}^{\mathrm{cl}}\left[s_{0}, s_{1}, s_{2}, s_{3}\right]$ denote the polynomial ring in indeterminates $k_{0}, k_{1}$ and $s_{\alpha}(0 \leq \alpha \leq 3)$, respectively. The Casimir relations (2.13) become the algebraic relations

$$
\begin{aligned}
& s_{1}^{2}+s_{2}^{2}+s_{3}^{2}=k_{0}, \\
& s_{0}^{2}+j_{1} s_{1}^{2}+j_{2} s_{2}^{2}+j_{3} s_{3}^{2}=k_{1}
\end{aligned}
$$

in $\mathbf{K}^{\mathrm{cl}}\left[s_{0}, s_{1}, s_{2}, s_{3}, k_{0}, k_{1}\right]$ :

$$
\mathbf{A}^{\mathrm{cl}} \simeq \mathbf{K}^{\mathrm{cl}}\left[s_{0}, s_{1}, s_{2}, s_{3}, k_{0}, k_{1}\right] /\left(s_{1}^{2}+s_{2}^{2}+s_{3}^{2}-k_{0}, s_{0}^{2}+j_{1} s_{1}^{2}+j_{2} s_{2}^{2}+j_{3} s_{3}^{2}-k_{1}\right) .
$$

This makes $\mathbf{A}^{\mathrm{cl}}$ an $\mathbf{F}^{\mathrm{cl}}$-algebra. The algebra $\mathbf{A}^{\mathrm{cl}}$ is graded as well: $\mathbf{A}^{\mathrm{cl}}=\oplus_{n=0}^{\infty} \mathbf{A}_{n}^{\mathrm{cl}}$. Let $\left(\mathbf{A}^{\mathrm{cl}}\right)_{n}^{\prime} \subset \mathbf{A}_{n}^{\mathrm{cl}}$ be the limit (in the appropriate Grassmannian such that we consider $s_{\alpha}$ as commutative variables) of $\mathbf{A}_{n}^{\prime}$ as $\varepsilon \rightarrow 0$. Then, we see $\sum_{\alpha=0}^{3}\left\{s_{\alpha}, \mathbf{A}_{n-1}^{\mathrm{cl}}\right\} \subset$ $\left(\mathbf{A}^{\mathrm{cl}}\right)_{n}^{\prime}$ from (A.6). In order to show the spanning property of $\left(m_{i}\right)_{1 \leq i \leq 7}$ in $\mathbf{H}$, it is therefore sufficient to show the spanning property for

$$
\left(m_{i}^{\mathrm{cl}}\right)_{1 \leq i \leq 7}=\left(1, s_{0}, s_{1}, s_{2}, s_{3}, s_{0}^{2}, s_{3}^{2}\right) .
$$

in $\mathbf{H}^{\mathrm{cl}}=\oplus_{n=0}^{\infty} \mathbf{H}_{n}^{\mathrm{cl}}$, where

$$
\mathbf{H}_{n}^{\mathrm{cl}}=\mathbf{A}_{n}^{\mathrm{cl}} / \sum_{\alpha=0}^{3}\left\{s_{\alpha}, \mathbf{A}_{n-1}^{\mathrm{cl}}\right\} .
$$

In conclusion, Theorem A.1 follows from

Proposition A.2. The $\mathbf{F}^{\mathrm{cl}}$-module $\mathbf{H}^{\mathrm{cl}}$ is a rank 7 free module generated by the monomials (A.11).

Proof. The $\mathbf{F}^{\mathrm{cl}}$-linear independence of $m_{i}^{\mathrm{cl}}$ follows from the same argument as in the quantum case. In place of trace on $\mathcal{V}^{(k)}$ we use the non-degenerate pairing between cycles and cocycles given in Appendix B.

We prove the spanning property. Define

$$
\begin{aligned}
\nabla_{0} & =j_{2,3} s_{2} s_{3} \frac{\partial}{\partial s_{1}}+j_{3,1} s_{3} s_{1} \frac{\partial}{\partial s_{2}}+j_{1,2} s_{1} s_{2} \frac{\partial}{\partial s_{3}} \\
\nabla_{a} & =-j_{b, c} s_{b} s_{c} \frac{\partial}{\partial s_{0}}+s_{0} s_{c} \frac{\partial}{\partial s_{b}}-s_{0} s_{b} \frac{\partial}{\partial s_{c}}
\end{aligned}
$$

where $(a, b, c)=(1,2,3),(2,3,1),(3,1,2)$. We have $\nabla_{\alpha} P=\frac{1}{2}\left\{s_{\alpha}, P\right\}$. These are $\mathbf{F}^{\mathrm{cl}}$-linear.

We want to show that modulo $\sum_{\alpha=0}^{3} \nabla_{\alpha} \mathbf{A}^{\text {cl }}$ any monomial $s_{0}^{m_{0}} s_{1}^{m_{1}} s_{2}^{m_{2}} s_{3}^{m_{3}}$ can be reduced to an element in $\mathbf{F}^{\mathrm{cl}} \cdot 1+\sum_{\alpha=0}^{3} \mathbf{F}^{\mathrm{cl}} \cdot s_{\alpha}+\sum_{\alpha=0}^{3} \mathbf{F}^{\mathrm{cl}} \cdot s_{\alpha}^{2}$. Set

$$
\begin{aligned}
\mathbf{A}^{\mathrm{cl}[-1]} & =\sum_{0 \leq \alpha<\beta \leq 3} s_{\alpha} s_{\beta} \mathbf{A}^{\mathrm{cl}}, \\
\mathbf{H}^{\mathrm{cl}[-1]} & =\mathbf{A}^{\mathrm{cl}[-1]} / \sum_{\alpha=0}^{3} \nabla_{\alpha} \mathbf{A}^{\mathrm{cl}} .
\end{aligned}
$$


We also denote $\mathbf{H}^{\mathrm{cl}[0]}=\mathbf{H}^{\mathrm{cl}}$ and $\mathbf{A}^{\mathrm{cl}[0]}=\mathbf{A}^{\mathrm{cl}}$.

Since

$$
\mathbf{A}^{\mathrm{cl}}=\left(\sum_{\alpha=0}^{3} \sum_{n=0}^{\infty} \mathbf{K}^{\mathrm{cl}} s_{\alpha}^{n}\right) \oplus \mathbf{A}^{\mathrm{cl}[-1]},
$$

by using (A.9) and (A.10) the above statement follows from the following:

$$
\mathbf{H}^{\mathrm{cl}[-1]}=\mathbf{F}^{\mathrm{cl}} k_{0} s_{0}+\sum_{a=1}^{3} \mathbf{F}^{\mathrm{cl}}\left(k_{1}-j_{a} k_{0}\right) s_{a}+\sum_{a=1}^{3} \mathbf{F}^{\mathrm{cl}}\left(k_{1}-j_{a} k_{0}\right) s_{a}^{2} .
$$

Note that

$$
k_{1}-j_{a} k_{0}=s_{0}^{2}+j_{b, a} s_{b}^{2}+j_{c, a} s_{c}^{2}
$$

and therefore we have $k_{0} s_{0},\left(k_{1}-j_{a} k_{0}\right) s_{a},\left(k_{1}-j_{a} k_{0}\right) s_{a}^{2} \in \mathbf{A}^{\mathrm{cl}[-1]}$.

Let us prove (A.13). Suppose that a monomial $m=s_{0}^{n_{0}} s_{1}^{n_{1}} s_{2}^{n_{2}} s_{3}^{n_{3}} \in \mathbf{H}^{\mathrm{cl}[-1]}$ is such that $\sharp\left\{a \mid n_{a} \in 2 \mathbb{Z}+1\right\} \geq 2$ : e.g., $n_{0}, n_{1} \in 2 \mathbb{Z}+1$. By using the Casimir relations (A.9) and (A.10) we can replace $s_{0}^{2}$ and $s_{1}^{2}$ with $s_{2}^{2}$ and $s_{3}^{2}$. Therefore, we have

$$
m \in s_{0} s_{1} \mathbf{F}^{\mathrm{cl}}\left[s_{2}, s_{3}\right] .
$$

Since

$$
\nabla_{2}\left(s_{2}^{j} s_{3}^{k}\right)=k s_{0} s_{1} s_{2}^{j} s_{3}^{k-1}
$$

we have

$$
m=0 \text {. }
$$

Next consider the case $\sharp\left\{a \mid n_{a} \in 2 \mathbb{Z}+1\right\}=1$. Suppose $n_{0} \in 2 \mathbb{Z}+1$. Then, we have

$$
m \in s_{0} s_{2}^{2} \mathbf{F}^{\mathrm{cl}}\left[s_{2}^{2}, s_{3}^{2}\right]+s_{0} s_{3}^{2} \mathbf{F}^{\mathrm{cl}}\left[s_{2}^{2}, s_{3}^{2}\right]
$$

Since

$$
\nabla_{1}\left(s_{1}^{i} s_{2}^{l} s_{3}^{k}\right)=l s_{0} s_{1}^{i} s_{2}^{l-1} s_{3}^{k+1}-k s_{0} s_{1}^{i} s_{2}^{l+1} s_{3}^{k-1},
$$

the monomial $m$ belongs to $\mathbf{K}^{\mathrm{cl}} s_{0} s_{2}^{2 j} \subset \mathbf{H}^{\mathrm{cl}[-1]}$ where $2 j+1=n_{0}+n_{1}+n_{2}+n_{3}$. Similarly, we see that

$$
k_{0}^{j} s_{0} \in \mathbf{K}^{\mathrm{cl}} s_{0} s_{2}^{2 j} .
$$

Since $k_{0}^{j} s_{0}$ is a non-zero element in $\mathbf{H}^{\mathrm{cl}[-1]}$, we have

$$
m \in \mathbf{K}^{\mathrm{cl}} k_{0}^{j} s_{0}
$$

The case $n_{a} \in 2 \mathbb{Z}+1(a=1,2,3)$ is similar.

The remaining case is $n_{0}, n_{1}, n_{2}, n_{3} \in 2 \mathbb{Z}$ and $\sharp\left\{a \mid n_{a}>0\right\} \geq 2$. We have $\operatorname{deg} m \geq$ 4. Note that

$$
\mathbf{H}_{4}^{\mathrm{cl}[-1]}=\sum_{0 \leq i<j \leq 3} \mathbf{K}^{\mathrm{cl}} s_{i}^{2} s_{j}^{2}
$$


We have the following relations in $\mathbf{H}_{4}^{\mathrm{cl}[-1]}$ :

$$
\begin{aligned}
& \nabla_{1}\left(s_{0} s_{2} s_{3}\right)=-j_{2,3} s_{2}^{2} s_{3}^{2}+s_{0}^{2} s_{3}^{2}-s_{0}^{2} s_{2}^{2}, \\
& \nabla_{2}\left(s_{0} s_{3} s_{1}\right)=-j_{3,1} s_{3}^{2} s_{1}^{2}+s_{0}^{2} s_{1}^{2}-s_{0}^{2} s_{3}^{2}, \\
& \nabla_{3}\left(s_{0} s_{1} s_{2}\right)=-j_{1,2} s_{1}^{2} s_{2}^{2}+s_{0}^{2} s_{2}^{2}-s_{0}^{2} s_{1}^{2} .
\end{aligned}
$$

Therefore, we have $\operatorname{dim}_{\mathbf{K}^{\mathrm{cl}}} \mathbf{H}_{4}^{\mathrm{cl}[-1]} \leq 3$. On the other hand $\left(k_{1}-j_{a} k_{0}\right) s_{a}^{2} \in \mathbf{H}_{4}^{\mathrm{cl}[-1]}$ for $a=1,2,3$, and they are $\mathbf{K}^{\mathrm{cl}}$-linearly independent. Therefore, we have

$$
\mathbf{H}_{4}^{\mathrm{cl}[-1]}=\oplus_{a=1}^{3} \mathbf{K}^{\mathrm{cl}}\left(k_{1}-j_{a} k_{0}\right) s_{a}^{2} .
$$

Observe that for $(a, b, c)=(1,2,3),(2,3,1),(3,1,2)$ we have

$$
\begin{gathered}
\nabla_{a}\left(s_{0}^{n_{0}+1} s_{1}^{n_{1}} s_{2}^{n_{2}} s_{3}^{n_{3}}\right)=-\left(n_{0}+1\right) j_{b, c} s_{0}^{n_{0}} s_{a}^{n_{a}} s_{b}^{n_{b}+1} s_{c}^{n_{c}+1} \\
\quad+n_{b} s_{0}^{n_{0}+2} s_{a}^{n_{a}} s_{b}^{n_{b}-1} s_{c}^{n_{c}+1}-n_{c} s_{0}^{n_{0}+2} s_{a}^{n_{a}} s_{b}^{n_{b}+1} s_{c}^{n_{c}-1} .
\end{gathered}
$$

We can increase the power in $s_{0}$ by rewriting $s_{0}^{n_{0}} s_{a}^{n_{a}} s_{b}^{n_{b}+1} s_{c}^{n_{c}+1}$ in terms of $s_{0}^{n_{0}+2} s_{a}^{n_{a}} s_{b}^{n_{b}-1} s_{c}^{n_{c}+1}$ and $s_{0}^{n_{0}+2} s_{a}^{n_{a}} s_{b}^{n_{b}+1} s_{c}^{n_{c}-1}$. Thus, we see that the $\mathbf{K}^{\mathrm{cl}}$ vector space $\mathbf{H}_{2 j}^{\mathrm{cl}[-1]}(j \geq 3)$ is spanned by

$$
s_{0}^{2 d} s_{a}^{2(j-d)} \quad(a=1,2,3 ; 1 \leq d \leq j-1) .
$$

On the other hand, the space $\mathbf{H}_{2 j}^{\mathrm{cl}[-1]}$ contains $\mathbf{K}^{\mathrm{cl}}$-linearly independent elements

$$
k_{0}^{d} k_{1}^{j-d-2}\left(k_{1}-j_{a} k_{0}\right) s_{a}^{2} \quad(a=1,2,3 ; 0 \leq d \leq j-2) .
$$

Therefore, the elements (A.14) span $\mathbf{H}_{2 j}^{\mathrm{cl}[-1]}$.

In the rest of this appendix, we explain a mathematical background of Proposition A.2. which is the de Rham cohomology of the affine algebraic variety defined by the two quadrics (A.9) and (A.10). Although our proof is independent, the statement of Proposition A.2 is closely related to a result of K. Saito.

We set

$$
M=\left\{\left(j_{1}, j_{2}, j_{3}\right) \in \mathbb{C}^{3} \mid j_{a} \neq j_{b} \text { for } a \neq b\right\}
$$

Let

$$
\varphi: X=\mathbb{C}^{4} \times M \rightarrow Y=\mathbb{C}^{2} \times M
$$

be the mapping such that $\varphi=\left(\varphi_{1}, \ldots, \varphi_{5}\right)$ and for $(\mathbf{s}, \mathbf{j})=\left(s_{0}, s_{1}, s_{2}, s_{3}, j_{1}, j_{2}, j_{3}\right) \in$ $\mathbb{C}^{4} \times M$ we have

$$
\begin{aligned}
& \varphi_{1}(\mathbf{s}, \mathbf{j})=s_{1}^{2}+s_{2}^{2}+s_{3}^{2}, \\
& \varphi_{2}(\mathbf{s}, \mathbf{j})=s_{0}^{2}+j_{1} s_{1}^{2}+j_{2} s_{2}^{2}+j_{3} s_{3}^{2}, \\
& \varphi_{3}(\mathbf{s}, \mathbf{j})=j_{1}, \\
& \varphi_{4}(\mathbf{s}, \mathbf{j})=j_{2}, \\
& \varphi_{5}(\mathbf{s}, \mathbf{j})=j_{3} .
\end{aligned}
$$

The critical set $C \subset \mathbb{C}^{4} \times M$ of this mapping is given by the equation

$$
d \varphi_{1} \wedge \cdots \wedge d \varphi_{5}=0 .
$$


We have

$$
C=\bigcup_{\alpha=0}^{3}\left\{(\mathbf{s}, \mathbf{j}) \mid s_{\beta}=0 \text { for } \beta \neq \alpha\right\} .
$$

We have the commutative diagram,

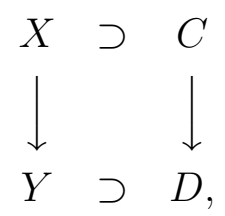

where the discriminant set $D$ is given by

$$
\begin{aligned}
D & =\left\{\left(k_{0}, k_{1}, j_{1}, j_{2}, j_{3}\right) \in \mathbb{C}^{2} \times M \mid \Delta\left(k_{0}, k_{1}, j_{1}, j_{2}, j_{3}\right)=0\right\} \\
\Delta & =k_{0} \prod_{a=1}^{3}\left(k_{1}-j_{a} k_{0}\right) .
\end{aligned}
$$

The inverse image $\varphi^{-1}(0)$ is called the simple elliptic singularity of type $\tilde{D}_{5}$ [29. If $y \in Y$ does not belong to $D$, the inverse image $X_{y}=\varphi^{-1}(y)$ is a non-singular affine complex surface and is called a smoothing of the singularity. The mapping

$$
\left.\varphi\right|_{X-\varphi^{-1}(D)}: X-\varphi^{-1}(D) \rightarrow Y-D
$$

is a locally topologically trivial fiber space, and the homology group is of rank 7: $H_{2}\left(X_{y}, \mathbb{Z}\right)=\mathbb{Z}^{7}$. In Appendix $\mathbb{B}$, we construct cycles in $H_{2}\left(X_{y}, \mathbb{Z}\right)$. (In [31], $\mathrm{K}$. Saito defined the extended affine root systems. The homology group $H_{2}\left(X_{y}, \mathbb{Z}\right)$ is isomorphic to $D_{5}^{(1,1)}$ in his classification. We have not identified our cycles in $D_{5}^{(1,1)}$.)

Let $\Omega_{X}^{p}$ be the sheaf of $\mathcal{O}_{X}$ modules consisting of germs of holomorphic $p$ forms on $X$, and $\Omega_{X / Y}^{p}$ the quotient sheaf

$$
\Omega_{X / Y}^{p}=\Omega_{X}^{p} / \sum_{i=1}^{5} d \varphi_{i} \wedge \Omega_{X}^{p-1} .
$$

The relative de Rham complex $\left(\Omega_{X / Y}^{\bullet}, d_{X / Y}\right)$ is defined by the commutative diagram

$$
\begin{aligned}
& d_{X / Y}: \Omega_{X / Y}^{p} \rightarrow \Omega_{X / Y}^{p+1}
\end{aligned}
$$

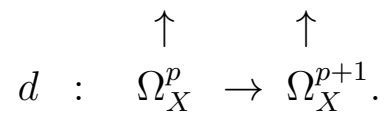

This is an exact sequence of $\mathcal{O}_{X}$ modules. The following is K. Saito's result [32].

Theorem A.3. The cohomology group

$$
H^{2}\left(\varphi_{*}\left(\Omega_{X / Y}^{\bullet}\right)\right)=\operatorname{Ker}\left(\varphi_{*}\left(\Omega_{X / Y}^{2}\right) \stackrel{d_{X / Y}}{\rightarrow} \varphi_{*}\left(\Omega_{X / Y}^{3}\right)\right) / \operatorname{Im}\left(\varphi_{*}\left(\Omega_{X / Y}^{1}\right) \stackrel{d_{X / Y}}{\rightarrow} \varphi_{*}\left(\Omega_{X / Y}^{2}\right)\right)
$$

is an $\mathcal{O}_{Y}$ locally free module of rank 7 .

We connect the above algebro-geometric setting to ours. The following proposition is a corollary to Theorem A.3. Here we give a proof in the line of this appendix without using Saito's result. 
Proposition A.4. Consider a complex of $\mathbf{F}^{\mathrm{cl}}$-modules:

$$
\begin{aligned}
Z^{p} & =\bigoplus_{0 \leq \alpha_{1}<\cdots<\alpha_{p} \leq 3} \mathbf{A}^{\mathrm{cl}} d s_{\alpha_{1}} \wedge \cdots \wedge d s_{\alpha_{p}} \\
\bar{Z}^{p} & =Z^{p} / \sum_{j=1,2} d \varphi_{j} \wedge Z^{p-1} .
\end{aligned}
$$

The cohomology group $\mathbf{H}^{\mathrm{cl}[-2]} \stackrel{\text { def }}{=} \operatorname{Ker}\left(\bar{Z}^{2} \stackrel{d}{\rightarrow} \bar{Z}^{3}\right) / \operatorname{Im}\left(\bar{Z}^{1} \stackrel{d}{\rightarrow} \bar{Z}^{2}\right)$ is a $\mathbf{F}^{\mathrm{cl}}$-free module of rank 7 , where the action of $k_{0}$ (resp., $\left.k_{1}\right)$ is given by the multiplication of $\varphi_{1}$ $\left(\right.$ resp., $\left.\varphi_{2}\right)$.

Proof. The key idea of the proof is to identify $\mathbf{A}^{\mathrm{cl}[-1]}$ with $\bar{Z}^{2}$.

Let us introduce a holomorphic section $\omega$ of the sheaf $\left.\Omega_{X / Y}^{2}\right|_{X-C}$.

$$
\begin{aligned}
\omega & =\frac{d s_{1} \wedge d s_{2}}{s_{0} s_{3}}=\frac{d s_{2} \wedge d s_{3}}{s_{0} s_{1}}=\frac{d s_{3} \wedge d s_{1}}{s_{0} s_{2}} \\
& =\frac{d s_{0} \wedge d s_{1}}{j_{2,3} s_{2} s_{3}}=\frac{d s_{0} \wedge d s_{2}}{j_{3,1} s_{3} s_{1}}=\frac{d s_{0} \wedge d s_{3}}{j_{1,2} s_{1} s_{2}}
\end{aligned}
$$

Consider the $\mathbf{F}^{\mathrm{cl}}$-module

$$
\tilde{\mathbf{A}}^{\mathrm{cl}} \stackrel{\text { def }}{=} \mathbf{K}^{\mathrm{cl}} \otimes_{\mathbb{C}\left[j_{1}, j_{2}, j_{3}\right]} \mathbb{C}\left[s_{0}, s_{1}, s_{2}, s_{3}, j_{1}, j_{2}, j_{3}\right] \omega
$$

We have a canonical isomorphism of $\mathbf{F}^{\mathrm{cl}}$-modules

$$
\mathbf{A}^{\mathrm{cl}} \simeq \tilde{\mathbf{A}}^{\mathrm{cl}}
$$

sending $P \in \mathbf{A}^{\text {cl }}$ to $P \omega \in \tilde{\mathbf{A}}^{\text {cl }}$. It is easy to see that

$$
\mathbf{A}^{\mathrm{cl}[-1]} \omega=\bar{Z}^{2} .
$$

We have

$$
d\left(P d s_{\alpha}\right)=-\nabla_{\alpha}(P) \omega
$$

for $P \in \mathbf{A}^{\mathrm{cl}}$. From $\mathrm{A} .25$ we have

$$
\operatorname{Im}\left(\bar{Z}^{1} \stackrel{d}{\rightarrow} \bar{Z}^{2}\right)=\left(\sum_{\alpha=0}^{3} \nabla_{\alpha} \mathbf{A}^{\mathrm{cl}}\right) \omega
$$

We have already constructed the $\mathbf{F}^{\mathrm{cl}}$-bases of the modules $\mathbf{A}^{\mathrm{cl}} / \sum_{\alpha=0}^{3} \nabla_{\alpha} \mathbf{A}^{\mathrm{cl}}$ and $\mathbf{A}^{\mathrm{cl}[-1]} / \sum_{\alpha=0}^{3} \nabla_{\alpha} \mathbf{A}^{\mathrm{cl}}$. We will construct a basis of $\operatorname{Ker}\left(\bar{Z}^{2} \stackrel{d}{\rightarrow} \bar{Z}^{3}\right) / \operatorname{Im}\left(\bar{Z}^{1} \stackrel{d}{\rightarrow} \bar{Z}^{2}\right)$. First, observe that

$$
\bar{Z}^{3}=\bigoplus_{n=0}^{\infty} \mathbf{K}^{\mathrm{cl}} s_{0}^{n} d s_{1} \wedge d s_{2} \wedge d s_{3}+\sum_{a=1}^{3} \bigoplus_{n=0}^{\infty} \mathbf{K}^{\mathrm{cl}} s_{a}^{n} d s_{0} \wedge d s_{b} \wedge d s_{c}
$$


where $(a, b, c)=(1,2,3),(2,3,1),(3,1,2)$. From this we see that

$$
\begin{aligned}
& \operatorname{Ker}\left(\bar{Z}^{2} \stackrel{d}{\rightarrow} \bar{Z}^{3}\right)=\sum_{0 \leq \alpha<\beta<\gamma \leq 3} \mathbf{A}^{\mathrm{cl}} s_{\alpha} s_{\beta} s_{\gamma} \omega \oplus \sum_{\substack{m, n \geq 1 \\
m, n \neq 2}} \sum_{0 \leq \alpha<\beta \leq 3} \mathbf{K}^{\mathrm{cl}} s_{\alpha}^{m} s_{\beta}^{n} \omega \\
& \oplus \sum_{\substack{n \geq 1 \\
n \neq 2}}\left(\sum_{1 \leq a \neq b \leq 3} \mathbf{K}^{\mathrm{cl}}\left(s_{0}^{2}+j_{a b} s_{b}^{2}\right) s_{a}^{n} \omega+\sum_{1 \leq a \leq 2} \mathbf{K}^{\mathrm{cl}}\left(s_{a}^{2}-s_{a+1}^{2}\right) s_{0}^{n} \omega\right) \\
& \oplus \sum_{1 \leq a<b \leq 3} \mathbf{K}^{\mathrm{cl}}\left(j_{a b} s_{a}^{2} s_{b}^{2}+s_{0}^{2} s_{a}^{2}-s_{0}^{2} s_{b}^{2}\right) \omega .
\end{aligned}
$$

We know that the $\mathbf{F}^{\mathrm{cl}}$-module $\bar{Z}^{2} / \operatorname{Im}\left(\bar{Z}^{1} \stackrel{d}{\rightarrow} \bar{Z}^{2}\right)$ has the free generators $\kappa_{0}=k_{0} s_{0} \omega$, $\kappa_{a}=\left(k_{1}-j_{a} k_{0}\right) s_{a} \omega(a=1,2,3)$ and $\rho_{a}=\left(k_{1}-j_{a} k_{0}\right) s_{a}^{2} \omega(a=1,2,3)$. For each degree $e \geq 3$ and color $c=0,1,2,3$, we want to construct elements of degree $e$ and

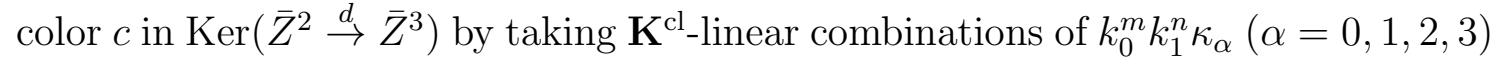
and $k_{0}^{m} k_{1}^{n} \rho_{a}(a=1,2,3)$. We set $\rho_{0}=k_{0} s_{0}^{2} \omega$. We have the relation $\rho_{0}=\rho_{1}+\rho_{2}+\rho_{3}$. A straightforward calculation shows that for $e=3,4$ we have none; for $e=5$ we find $\xi_{0}=k_{0} \kappa_{0}$ for color 0 and $\xi_{a}=\left(k_{1}-j_{a} k_{0}\right) \kappa_{a}$ for color $a=1,2,3$; for $e=6$ we find two color 0 elements:

$$
\begin{aligned}
& \xi_{4}=\left(k_{1}-j_{1} k_{0}\right) \rho_{1}-\left(k_{1}-j_{2} k_{0}\right) \rho_{2}-j_{1,2} k_{0} \rho_{0}, \\
& \xi_{5}=\left(k_{1}-j_{2} k_{0}\right) \rho_{2}-\left(k_{1}-j_{3} k_{0}\right) \rho_{3}-j_{2,3} k_{0} \rho_{0} .
\end{aligned}
$$

For $e=5,6$ the above elements span the degree $e$ cohomology classes. For $e=8$, we have 4 obvious elements $k_{i} \xi_{j}(i=0,1 ; j=4,5)$, and in addition, we find

$$
\xi_{6}=\frac{1}{4} k_{0}^{2} \sum_{a=1}^{3} \sum_{b \neq a} j_{a, b} \rho_{a}+k_{0} \sum_{a=1}^{3}\left(k_{1}-j_{a} k_{0}\right) \rho_{a} .
$$

These 5 elements span the degree 8 cohomology classes.

Finally, we show that $\xi_{a}(0 \leq a \leq 6)$ are the generators of the $\mathbf{F}^{\mathrm{cl}}$-module $\operatorname{Ker}\left(\bar{Z}^{2} \stackrel{d}{\rightarrow} \bar{Z}^{3}\right) / \operatorname{Im}\left(\bar{Z}^{1} \stackrel{d}{\rightarrow} \bar{Z}^{2}\right)$. For odd $e=2 n+3 \geq 7$, let us consider the color 0 case. The cases of other colors are similar. The degree $2 n+3$ and color 0 space in $\bar{Z}^{2} / \operatorname{Im}\left(\bar{Z}^{1} \stackrel{d}{\rightarrow} \bar{Z}^{2}\right)$ has the $\mathbf{K}^{\text {cl }}$-basis consisting of $n+1$ elements $k_{0}^{j} k_{1}^{(n-j)} \kappa_{0}$ $(0 \leq j \leq n)$. We have

$$
d\left(k_{1}^{n} \kappa_{0}\right)=3 s_{0}^{2 n} d s_{1} \wedge d s_{2} \wedge d s_{3} \in \bar{Z}^{3} .
$$

Therefore, the $\mathbf{K}^{\mathrm{cl}}$-dimension of the degree $2 n+3$ and color $\overline{0}$ subspace of $\operatorname{Ker}\left(\bar{Z}^{2} \stackrel{d}{\rightarrow}\right.$ $\left.\bar{Z}^{3}\right) / \operatorname{Im}\left(\bar{Z}^{1} \stackrel{d}{\rightarrow} \bar{Z}^{2}\right)$ is $n$. Since the $\mathbf{K}^{\mathrm{cl}}$-linearly independent elements $k_{0}^{j} k_{1}^{n-1-j} \xi_{0}$ $(0 \leq j \leq n-1)$ belongs to this subspace, they span the subspace.

For even $e=2 n+4 \geq 10$, the degree $2 n+4$ subspace of $\bar{Z}^{2} / \operatorname{Im}\left(\bar{Z}^{1} \stackrel{d}{\rightarrow} \bar{Z}^{2}\right)$ has the

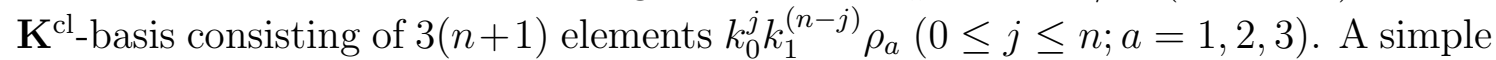
calculation shows that the elements $d\left(k_{1}^{n} \rho_{0}\right), d\left(k_{0}^{n} \rho_{a}\right)(a=1,2,3)$ are $\mathbf{K}^{\text {cl }}$-linearly independent in $\bar{Z}^{3}$. Therefore, the $\mathbf{K}^{\mathrm{cl}}$-dimension of the degree $2 n+4$ subspace of $\operatorname{Ker}\left(\bar{Z}^{2} \stackrel{d}{\rightarrow} \bar{Z}^{3}\right) / \operatorname{Im}\left(\bar{Z}^{1} \stackrel{d}{\rightarrow} \bar{Z}^{2}\right)$ is $3 n-1$. On the other hand, we obtain $3 n-1$ 
independent elements of the subspace from the degree 6 elements $\rho_{4}, \rho_{5}$ and the degree 8 element $\rho_{6}$. We conclude that they span the subspace.

We have explicitly constructed free bases for the spaces $\mathbf{H}^{\mathrm{cl}[0]} \supset \mathbf{H}^{\mathrm{cl}[-1]} \supset \mathbf{H}^{\mathrm{cl}[-2]}$. In [32], similar spaces are studied for hypersurface singularities $\tilde{E}_{6}, \tilde{E}_{7}, \tilde{E}_{8}$. The results in this appendix supplement some part of his construction in $\tilde{D}_{5}$, where the elliptic singularity is given by a complete intersection of two quadrics.

\section{Appendix B. CyCles AND InTEGRALS in the ClASSiCAl Limit}

In the classical limit, the defining relations of the Sklyanin algebra turns into (A.9), (A.10) which define an affine algebraic surface $\mathcal{S} \subset \mathbb{C}^{4}$. In this section, we study the classical limit of the functional $\operatorname{Tr}_{\lambda}$ and identify it with an integral over a cycle on $\mathcal{S}$.

Sklyanin's formulas for the representation (2.16) gives an explicit uniformization of $\mathcal{S}$. Consider the limit

$$
\eta \rightarrow 0, \quad \lambda \rightarrow \infty, \quad \eta \lambda \equiv \mu \text { finite. }
$$

In the right hand side of (2.16), we replace $\eta \partial_{u}$ by $-2 \pi i v$ where $v$ is the variable canonically conjugate to $u$,

$$
2 \pi\{v, u\}=1 \text {. }
$$

In the limit, the formulas for $s_{0}=S_{0}, s_{a}=\eta S_{a}(a=1,2,3)$ tend to

$$
s_{\alpha}(u, v)=c_{\alpha} \frac{\theta_{\alpha+1}(2 u-\mu) e^{-2 \pi i v}-\theta_{\alpha+1}(-2 u-\mu) e^{2 \pi i v}}{\theta_{1}(2 u)},
$$

where $(u, v) \in \mathbb{C}^{2}$ and

$$
c_{0}=\frac{1}{2}, \quad c_{a}=\sqrt{\varepsilon_{a}} \frac{\theta_{a+1}(0)}{2 \theta_{1}^{\prime}} \text { for } a=1,2,3 .
$$

Eq. (B.1) provides a parametrization of the surface (A.9), (A.10) with

$$
k_{0}=\left(\frac{\theta_{1}(\mu)}{\theta_{1}^{\prime}}\right)^{2}, \quad k_{1}=\left(\frac{\theta_{1}(\mu)}{\theta_{1}^{\prime}}\right)^{2} \partial^{2} \log \theta_{1}(\mu) .
$$

For definiteness, we consider the case $\tau \in i \mathbb{R}_{>0}, 0<\mu<1 / 2$. The functions $s_{\alpha}$ have common periods

$$
e_{1}=(1,0), \quad e_{2}=(0,1), \quad e_{3}=(\tau, 2 \mu),
$$

so $(u, v)$ should be regarded as variables on $\mathbb{C}^{2} /\left(\sum_{i=1}^{3} \mathbb{Z} e_{i}\right)$. We shall consider the fundamental domain:

$$
0 \leq \operatorname{Re}(u)<1, \quad 0 \leq \operatorname{Im}(u)<\frac{1}{i} \tau, \quad 0 \leq \operatorname{Re}(v)<1 .
$$

There are also pole divisors of $s_{\alpha}$ at $u=p_{i}$, where

$$
p_{0}=0, p_{1}=\frac{1}{2}, p_{2}=\frac{1+\tau}{2}, p_{3}=\frac{\tau}{2} \text {. }
$$

If we neglect these divisors, there are, obviously, three non-trivial 2-cycles which are tori with generators $\left(e_{1}, e_{3}\right),\left(e_{1}, e_{2}\right),\left(e_{2}, e_{3}\right)$. We denote them by $\gamma_{0}, \gamma_{1}, \gamma_{2}$. 
One can choose the tori $\gamma_{1}, \gamma_{2}$ so that they do not intersect with the pole divisors. As for $\gamma_{0}$, the first impression is that it hits the divisors. But actually one has to be very careful at this point. From the formulae $(\underline{B} .1)$ it follows that there are no singularities at (B.3) if $u=p_{0}, p_{1}$ and $v=0,1 / 2$, or $u=p_{2}, p_{3}$ and $v=\mu, \mu+1 / 2$. Hence the actual divisors are $\mathcal{D}=\mathcal{D}^{\prime} \cup \mathcal{D}^{\prime \prime}$, with

$$
\begin{aligned}
& \mathcal{D}^{\prime}=\cup_{i=0,1}\left\{\left(p_{i}, v\right) \mid 0 \leq \operatorname{Re}(v)<1, v \neq 0,1 / 2\right\} \\
& \mathcal{D}^{\prime \prime}=\cup_{i=2,3}\left\{\left(p_{i}, v\right) \mid 0 \leq \operatorname{Re}(v)<1, v \neq \mu, \mu+1 / 2\right\}
\end{aligned}
$$

This means, first of all, that we can modify $\gamma_{0}$ at $v=0$ and $v=\mu$ into a well-defined cycle without intersection with $\mathcal{D}$, as depicted in fig. 1 below.

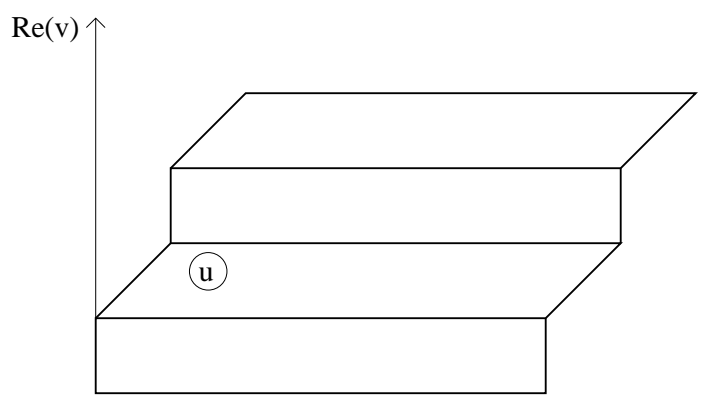

fig. 1

Now we have another possibility. We can draw spheres $\delta_{0}, \delta_{1}$ which have as south (resp. north) poles the points $\left(p_{0}, 0\right),\left(p_{1}, 0\right)$ (resp. $\left.\left(p_{0}, 1 / 2\right),\left(p_{1}, 1 / 2\right)\right)$. In the vicinity of these points they are parallel to the $u$-plane, and every section of it by the plane $\operatorname{Re}(v)=a$ for $0<a<1 / 2$ is a cycle around $p_{0}, p_{1}$ in the $u$-plane.

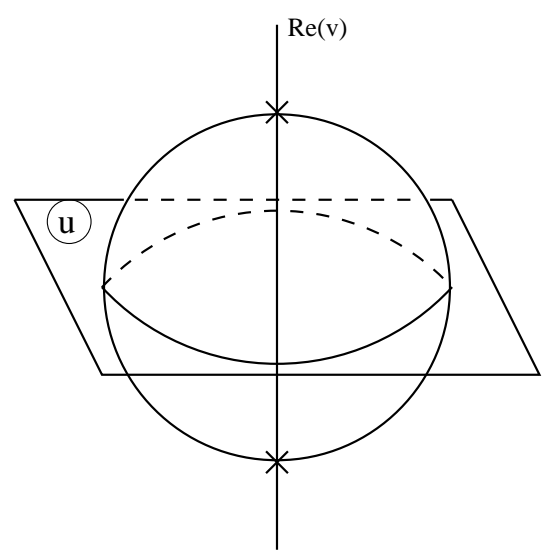

fig. 2

These spheres do not intersect $\mathcal{D}$. Similarly, we construct spheres $\delta_{2}, \delta_{3}$ which have as south (resp. north) poles the points $\left(p_{2}, \mu\right),\left(p_{3}, \mu\right)\left(\operatorname{resp} .\left(p_{2}, \mu+1 / 2\right),\left(p_{3}, \mu+1 / 2\right)\right)$.

The homology group $H_{2}(\mathcal{S}, \mathbb{Z}) \simeq \mathbb{Z}^{7}$ is known. We will see below that our cycles $\delta_{0}, \delta_{1}, \delta_{2}, \delta_{3}, \gamma_{0}, \gamma_{1}, \gamma_{2} \in H_{2}(\mathcal{S}, \mathbb{Z})$ give a linearly independent basis in $H_{2}(\mathcal{S}, \mathbb{C})$. 
Using our seven monomials we construct 2-forms

$$
\begin{aligned}
& \omega=-\frac{1}{4 \pi} \frac{d s_{1} \wedge d s_{2}}{s_{0} s_{3}}, \quad \omega_{0}=s_{0}^{2} \omega, \quad \omega_{3}=s_{3}^{2} \omega, \\
& \sigma_{\alpha}=s_{\alpha} \omega, \quad \alpha=0,1,2,3 .
\end{aligned}
$$

By direct computation we check that in Sklyanin's parameterization (B.1)

$$
\omega=d u \wedge d v .
$$

This formula allows us to calculate the integrals explicitly.

Let us start with the cycles $\delta_{\alpha}$. We obtain

$$
\int_{\delta_{\alpha}} \omega=\int_{\delta_{\alpha}} \omega_{\beta}=0, \quad \int_{\delta_{\alpha}} \sigma_{\beta}=2 \epsilon_{\alpha \beta} c_{\beta} \frac{\theta_{\beta+1}(-\mu)}{\theta_{1}^{\prime}},
$$

where $\epsilon_{\alpha \beta}$ are elements of the matrix:

$$
\epsilon=\left(\begin{array}{rrrr}
1 & 1 & 1 & 1 \\
1 & 1 & -1 & -1 \\
1 & -1 & 1 & -1 \\
1 & -1 & -1 & 1
\end{array}\right)
$$

Note, in particular, that

$$
\int_{\sum_{\alpha=0}^{3} \delta_{\alpha}} \sigma_{\beta}=0
$$

for $\beta=1,2,3$.

For the integrals over $\gamma_{k}(k=1,2)$, we find

$$
\begin{aligned}
& \int_{\gamma_{k}} \sigma_{\alpha}=0, \quad \int_{\gamma_{1}} \omega=1, \quad \int_{\gamma_{2}} \omega=\tau, \\
& \int_{\gamma_{1}} \omega_{\alpha}=-2\left(\frac{c_{\alpha} \theta_{\alpha+1}(\mu)}{\theta_{1}^{\prime}}\right)^{2} \partial^{2} \log \theta_{\alpha+1}(\mu), \\
& \int_{\gamma_{2}} \omega_{\alpha}=-2\left(\frac{c_{\alpha} \theta_{\alpha+1}(\mu)}{\theta_{1}^{\prime}}\right)^{2}\left(\tau \partial^{2} \log \theta_{\alpha+1}(\mu)+2 \pi i\right),
\end{aligned}
$$

For $\gamma_{0}$, we introduce

$$
\tilde{\gamma}_{0}=\gamma_{0}-2 \mu \gamma_{1}+\frac{1}{2} \sum_{\beta=0}^{3} \delta_{\beta}
$$

Then, we have

$$
\int_{\tilde{\gamma}_{0}} \omega=\int_{\tilde{\gamma}_{0}} \sigma_{\alpha}=0, \quad \int_{\tilde{\gamma}_{0}} \omega_{\alpha}=2\left(\frac{c_{\alpha} \theta_{\alpha+1}(\mu)}{\theta_{1}^{\prime}}\right)^{2} \partial \log \theta_{\alpha+1}^{2}(\mu),
$$


Using the above formulae we can calculate the determinant of the period-matrix as

$$
\operatorname{det}(\mathcal{P})=\text { Const } \cdot \theta_{1}(2 \mu)\left(\theta_{1}(\mu) \theta_{0}(\mu)\right)^{2} \frac{\partial}{\partial \mu} \log \left(\frac{\theta_{1}(\mu)}{\theta_{0}(\mu)}\right),
$$

whence we conclude that for generic $\mu$ the period matrix is non-degenerate.

Comparison with the quantum formulae (2.20), (2.21) shows the exceptional role of the cycle $\gamma_{0}$ as $\eta \rightarrow 0$,

$$
\begin{aligned}
& \operatorname{Tr}_{\lambda}\left(S_{\alpha}\right) \sim \frac{1}{2 \eta} \int_{\gamma_{0}} s_{\alpha} \omega \\
& \operatorname{Tr}_{\lambda}\left(S_{\alpha}^{2}\right) \sim \frac{1}{2 \eta} \int_{\gamma_{0}} s_{\alpha}^{2} \omega \times \begin{cases}1 & (\alpha=0), \\
\frac{1}{\eta^{2}} & (\alpha=1,2,3) .\end{cases}
\end{aligned}
$$

Actually, classical limits of all the elements of $F$ in (2.22)-(2.24) can be found among integrals over the cycles $\gamma_{0}, \gamma_{1}, \gamma_{2}$. This fact played an important heuristic role in the calculation of traces.

\section{Appendix C. Technical Lemmas}

In this Appendix, we collect some technical matters related to $\operatorname{Tr}_{\lambda}$. The first is the color conservation for $\operatorname{Tr}_{\lambda}$.

Lemma C.1. If $A \in \mathcal{A}^{(m, n)}$, then

$$
\operatorname{Tr}_{\lambda} A=0 \quad \text { unless }(m, n)=(0,0) .
$$

Proof. Set

$$
\varphi_{1}(f)(u):=f\left(u+\frac{\tau}{2}\right) e^{2 \pi i k u}, \quad \varphi_{2}(f)(u):=f\left(u+\frac{1}{2}\right) .
$$

One verifies easily that $\varphi_{1}, \varphi_{2} \in \operatorname{End}\left(\mathcal{V}^{(k)}\right)$ and

$$
\begin{aligned}
& \varphi_{1} \circ \pi^{(k)}\left(S_{\alpha}\right) \circ \varphi_{1}^{-1}=(-1)^{\bar{\alpha}_{1}} \pi^{(k)}\left(S_{\alpha}\right), \\
& \varphi_{2} \circ \pi^{(k)}\left(S_{\alpha}\right) \circ \varphi_{2}^{-1}=(-1)^{\bar{\alpha}_{2}} \pi^{(k)}\left(S_{\alpha}\right),
\end{aligned}
$$

where $\bar{\alpha}=\left(\bar{\alpha}_{1}, \bar{\alpha}_{2}\right) \in \mathbb{Z}_{2} \times \mathbb{Z}_{2}$. It follows that

$$
\operatorname{tr}_{V^{(k)}} \pi^{(k)}(A)=(-1)^{m} \operatorname{tr}_{V^{(k)}} \pi^{(k)}(A)=(-1)^{n} \operatorname{tr}_{V^{(k)}} \pi^{(k)}(A),
$$

whence the lemma.

The next Lemma is concerned about the uniqueness of the representation of $\operatorname{Tr}_{\lambda}$.

Lemma C.2. Assume $\operatorname{Im} \eta, \operatorname{Im} \tau>0, \eta \notin \mathbb{Q}+\mathbb{Q} \tau$. Let $g_{i}(i=1,2,3)$ be elliptic functions with periods 1 and $\tau$, and set $f(u)=\zeta(u) g_{1}(u)+u g_{2}(u)+g_{3}(u)$, where $\zeta(u)=-(1 / 2 \pi i) \theta_{1}^{\prime}(u) / \theta_{1}(u)$. If there exists an $N>0$ such that $f(k \eta)=0$ holds for all integers $k>N$, then we have $g_{i}(u) \equiv 0(i=1,2,3)$. 
Proof. We divide into three cases, (i) $g_{1}(u)=g_{2}(u)=0$, (ii) $g_{1}(u) \not \equiv 0$ and $g_{2}(u)=0$, (iii) $g_{2}(u) \not \equiv 0$.

Since $K=\{k \eta \mid k>N\}$ has accumulation points, the assertion is evident in case (i). Let us show that (ii), (iii) lead to contradictions.

In case (ii), considering $f(u) / g_{1}(u)$ we may assume $g_{1}(u)=1$. Then $f(u+\tau)=$ $f(u)+1$. Choose a point $u_{0} \in \mathbb{C} \backslash(K \cup L)$ which is not a pole of $f(u)$. One can find a sequence of integers $k_{1}<k_{2}<\cdots, k_{n} \rightarrow \infty$, such that $k_{n} \eta$ tends to $u_{0}$ in $\mathbb{C} / L$. Since $\operatorname{Im} \eta>0$, if we write $k_{n} \eta=a_{n}+b_{n} \tau\left(a_{n}, b_{n} \in \mathbb{R}\right)$, then the integer part of $b_{n}$ diverges. Therefore $f\left(k_{n} \eta\right)$ diverges as $n \rightarrow \infty$, which contradicts to the assumption $f\left(k_{n} \eta\right)=0$.

In case (iii), we may assume $g_{2}(u)=1$. Set $F(u)=f(u+\eta)-f(u)$. We have $F(k \eta)=0(k>N), F(u+1)=F(u)$, and $F(u+\tau)-F(u)=g_{1}(u+\eta)-g_{1}(u)$. Hence $F(u)=G(u)+\zeta(u)\left(g_{1}(u+\eta)-g_{1}(u)\right)$ with some elliptic function $G(u)$. From cases (i), (ii) we conclude that $F(u)=0$. In particular $g_{1}$ is a constant. Considering $f^{\prime}(u+\eta)=f^{\prime}(u)$, we find that $f(u)$ is a linear function. Clearly this is impossible.

Let us sketch the derivation of the formulas for $\operatorname{Tr}_{\lambda} S_{\alpha}, \operatorname{Tr}_{\lambda} S_{\alpha}^{2}$. We have the standard functional relation

$$
t^{(1)}\left(t-\frac{k}{2} \eta\right) t^{(k)}\left(t+\frac{1}{2} \eta\right)=\phi(t+\eta) t^{(k+1)}(t)+\phi(t) t^{(k-1)}(t+\eta)
$$

for the transfer matrices

$$
t^{(k)}(t):=\operatorname{tr}_{\mathcal{V}^{(k)}}\left(r_{a, N}^{(k, 1)}\left(t-t_{N}\right) \cdots r_{a, 1}^{(k, 1)}\left(t-t_{1}\right)\right) \quad \in \operatorname{End}\left(V^{\otimes N}\right),
$$

where $r^{(k, 1)}(t):=\left(\pi^{(k)} \otimes \mathrm{id}\right) L(t)$ and $\phi(t)=\prod_{j=1}^{N}\left[t-t_{j}-(k / 2) \eta\right]$. Choosing $N=$ $1, t_{1}=0$ and applying (C.1), we easily find (2.20). The same method is applicable for (2.21). We find it slightly simpler to use the difference equation for the matrices $\widehat{X}_{a, 2}^{(1,2)}$.

\section{Appendix D. Transformation properties of $\widehat{X}_{n}^{(i, j)}$}

Let us study the transformation properties of $\widehat{X}_{n}^{(i, j)}$ with respect to the shift of variables by half periods. For that purpose we exploit the order 4 automorphisms $\iota^{1}, \iota^{3}$ of the Sklyanin algebra $\mathcal{A}$ given by

$$
\begin{aligned}
& \iota^{1}\left(S_{0}\right)=-\frac{\theta_{1}(\eta)}{\theta_{2}(\eta)} S_{1}, \iota^{1}\left(S_{1}\right)=\frac{\theta_{2}(\eta)}{\theta_{1}(\eta)} S_{0}, \iota^{1}\left(S_{2}\right)=i \frac{\theta_{3}(\eta)}{\theta_{0}(\eta)} S_{3}, \iota^{1}\left(S_{3}\right)=-i \frac{\theta_{0}(\eta)}{\theta_{3}(\eta)} S_{2}, \\
& \iota^{3}\left(S_{0}\right)=\frac{\theta_{1}(\eta)}{\theta_{0}(\eta)} S_{3}, \iota^{3}\left(S_{1}\right)=\frac{\theta_{2}(\eta)}{\theta_{3}(\eta)} S_{2}, \iota^{3}\left(S_{2}\right)=-\frac{\theta_{3}(\eta)}{\theta_{2}(\eta)} S_{1}, \iota^{3}\left(S_{3}\right)=\frac{\theta_{0}(\eta)}{\theta_{1}(\eta)} S_{0} .
\end{aligned}
$$

In terms of the $L$-operator they can be written as

$$
\begin{aligned}
& \iota^{1}(L(t))=L\left(t+\frac{1}{4}\right) \sigma^{1}, \\
& \iota^{3}(L(t))=L\left(t+\frac{\tau}{4}\right) \sigma^{3} \times(-i) e^{\pi i(2 t+\eta+\tau / 4)} .
\end{aligned}
$$


Lemma D.1. Let $A \in \mathcal{A}_{n}$ be an element of the Sklyanin algebra of even degree $n$, and let $\theta_{1}(t)^{-n} \operatorname{Tr}_{t / \eta} A=g_{A, 1}(t)-(t / \eta) g_{A, 2}(t)$ where $g_{A, 1}, g_{A, 2}$ are as in (2.18). Then

$$
\begin{aligned}
& \theta_{1}(t)^{-n} \operatorname{Tr}_{\frac{t}{\eta}+\frac{1}{2 \eta}} l^{1}(A)=g_{A, 1}(t)-\left(\frac{t}{\eta}+\frac{1}{2 \eta}\right) g_{A, 2}(t), \\
& \theta_{1}(t)^{-n} \operatorname{Tr}_{\frac{t}{\eta}+\frac{\tau}{2 \eta}} l^{3}(A) \\
& \quad=\left(g_{A, 1}(t)+\frac{1}{2} g_{A, 3}(t)-\left(\frac{t}{\eta}+\frac{\tau}{2 \eta}\right) g_{A, 2}(t)\right) \times\left(-e^{-\pi i(\tau / 2+2 t)}\right)^{n / 2},
\end{aligned}
$$

where $g_{A, 3}(t)=g_{A, 1}(t+\tau)-g_{A, 1}(t)$ is an elliptic function.

Proof. In view of Theorem A.1, it is enough to consider elements $A$ of the form $m \cdot S_{\alpha}^{2}$, where $m$ is a polynomial in $K_{0}, K_{2}$ of degree $(n-2) / 2$. For $n=2$, the assertion can be verified from the explicit formula (2.21).

Let $I_{t}$ denote the two-sided ideal of $\mathcal{A}$ generated by $K_{0}-4 \theta_{1}(t)^{2} / \theta_{1}(2 \eta)^{2}, K_{2}-$ $4 \theta_{1}(t+\eta) \theta_{1}(t-\eta) / \theta_{1}(2 \eta)^{2}$, and let $\varpi_{t}: \mathcal{A} \rightarrow \mathcal{A} / I_{t}$ be the projection. From (2.15) and (2.17) we have

$$
\varpi_{t}\left(L_{1}\left(\frac{s}{2}\right) L_{2}\left(\frac{s}{2}-\eta\right)\right) \mathcal{P}_{12}^{-}=-\frac{\theta_{1}(t-s) \theta_{1}(t+s)}{\theta_{1}^{2}(2 \eta)} \mathcal{P}_{12}^{-} .
$$

Along with (D.1) and (D.2) it follows that for $i=0,2$

$$
\begin{aligned}
& \varpi_{t+1 / 2}\left(\iota^{1}\left(K_{i}\right)\right)=\varpi_{t}\left(K_{i}\right), \\
& \varpi_{t+\tau / 2}\left(\iota^{3}\left(K_{i}\right)\right)=\varpi_{t}\left(K_{i}\right) \times(-1) e^{-\pi i(\tau / 2+2 t)} .
\end{aligned}
$$

The Lemma follows from these relations.

Proposition D.2. The $\widehat{X}_{a, n}^{(i, j)}$ obey the following transformation laws.

$$
\begin{aligned}
& \sigma_{\bar{k}}^{1} \sigma_{k}^{1} \widehat{X}_{a, n}^{(i, j)}\left(\cdots, t_{k}+\frac{1}{2}, \cdots\right) \\
& \quad=\widehat{X}_{a, n}^{(i, j)}\left(\cdots, t_{k}, \cdots\right) \times \begin{cases}\sigma_{k}^{1} \sigma_{\bar{k}}^{1} & (k \neq i, j), \\
(-1)^{n-1} \prod_{p(\neq i, j)} \sigma_{p}^{1} \sigma_{\bar{p}}^{1} & (k=i, j),\end{cases}
\end{aligned}
$$

$$
\begin{aligned}
& \sigma_{\bar{k}}^{3} \sigma_{k}^{3} \widehat{X}_{a, n}^{(i, j)}\left(\cdots, t_{k}+\frac{\tau}{2}, \cdots\right)(k \neq i, j), \\
& \quad= \begin{cases}\widehat{X}_{a, n}^{(i, j)}\left(\cdots, t_{k}, \cdots\right) \sigma_{k}^{3} \sigma_{\bar{k}}^{3} \\
\left(\widehat{X}_{a, n}^{(i, j)}\left(\cdots, t_{k}, \cdots\right) \pm \frac{1}{2} \delta_{a 1} \widehat{X}_{3, n}^{(i, j)}\left(\cdots, t_{k}, \cdots\right)\right) \times(-1)^{n-1} \prod_{p(\neq i, j)} \sigma_{\bar{p}}^{3} \sigma_{p}^{3}, & (k=i, j) .\end{cases}
\end{aligned}
$$

In the last line the upper (resp. lower) sign in chosen for $k=i$ (resp. $k=j)$. In particular, $\widehat{X}_{2, n}^{(i, j)}, \widehat{X}_{3, n}^{(i, j)}$ are elliptic functions of $t_{1}, \cdots, t_{n}$ with periods $1, \tau$. 
Proof. It is enough to prove the case $(i, j)=(1,2)$. If $k \neq 1,2$, this is a simple consequence of the transformation law of the $L$-operator

$$
\begin{aligned}
& L\left(t+\frac{1}{2}\right)=-\sigma^{1} L(t) \sigma^{1}, \\
& L\left(t+\frac{\tau}{2}\right)=-\sigma^{3} L(t) \sigma^{3} \times e^{-2 \pi i(2 t+\eta+\tau / 2)} .
\end{aligned}
$$

Consider the case $k=1$. Using the automorphism $\iota^{1}$ we have

$$
\begin{aligned}
& \widehat{X}_{n}^{(1,2)}\left(t_{1}+\frac{1}{2}, \cdots\right) \times\left[t_{12}+1 / 2\right] \prod_{p=3}^{n}\left[t_{1 p}+1 / 2\right]\left[t_{2 p}\right] \\
& =\operatorname{Tr}_{\frac{t_{12}}{\eta}+\frac{1}{2 \eta}}\left(T^{[1]}\left(\frac{t_{1}+t_{2}}{2}+\frac{1}{4} ; t_{1}+\frac{1}{2}, \cdots, t_{n}\right)\right) P_{12} \mathcal{P}_{1 \overline{1}}^{-} \mathcal{P}_{2 \overline{2}}^{-} \\
& =\operatorname{Tr}_{\frac{t_{12}}{\eta}+\frac{1}{2 \eta}} \iota^{1}\left(T^{[1]}\left(\frac{t_{1}+t_{2}}{2} ; t_{1}, \cdots, t_{n}\right)\right) \prod_{p=2}^{n} \sigma_{\bar{p}}^{1} \sigma_{p}^{1} P_{12} \mathcal{P}_{1 \overline{1}}^{-} \mathcal{P}_{2 \overline{2}}^{-} \\
& =\sigma_{\overline{1}}^{1} \sigma_{1}^{1} \operatorname{Tr}_{\frac{t_{12}}{\eta}+\frac{1}{2 \eta}} \iota^{1}\left(T^{[1]}\left(\frac{t_{1}+t_{2}}{2} ; t_{1}, \cdots, t_{n}\right)\right) P_{12} \mathcal{P}_{1 \overline{1}}^{-} \mathcal{P}_{2 \overline{2}}^{-} \prod_{p=3}^{n} \sigma_{\bar{p}}^{1} \sigma_{p}^{1} .
\end{aligned}
$$

Applying Lemma D.1 we obtain (D.3) with $k=1$. Eq. (D.4 is shown similarly. The case $k=2$ can be obtained by using the translation invariance.

Acknowledgments. Research of HB is supported by the RFFI grant \#04-01-00352. Research of MJ is supported by the Grant-in-Aid for Scientific Research B2-16340033 and A2-14204012. Research of TM is supported by the Grant-in-Aid for Scientific Research A1-13304010. Research of FS is supported by INTAS grant \#03-513350 and by EC networks "EUCLID", contract number HPRN-CT-2002-00325 and "ENIGMA", contract number MRTN-CT-2004-5652. Research of YT is supported by University of Tsukuba Research Project. This work was also supported by the grant of 21st Century COE Program at Graduate School of Mathematical Sciences, the University of Tokyo, and at RIMS, Kyoto University.

MJ, TM and YT are grateful to K. Saito for explaining his theory of simpleelliptic singularities. MJ thanks V. Mangazeev, A. Zabrodin, A. Mironov, C. Korff and R. Weston for discussions. HB thanks M. Lashkevich for tight cooperation in numerical checking. He also thanks F. Göhmann, A. Klümper and K. Fabricius for discussions. Last but not least, HB and FS wish to thank the University of Tokyo for the invitation and hospitality where the present work was started, and HB, JM, TM and YT to Université Paris VI for hospitality, where it was completed.

\section{REFERENCES}

[1] R. Baxter, Exactly solved models in statistical mechanics, Academic Press, London 1982.

[2] R. Baxter, Solvable eight-vertex model on an arbitrary planar lattice, Phil. Trans. Royal Soc. London, 289 (1978) 315-346. 
[3] H. Boos, M. Jimbo, T. Miwa, F. Smirnov and Y. Takeyama, A recursion formula for the correlation functions of an inhomogeneous XXX model, hep-th/0405044 to appear in Algebra and Analysis.

[4] H. Boos, M. Jimbo, T. Miwa, F. Smirnov and Y. Takeyama, Reduced qKZ equation and correlation functions of the XXZ model, hep-th/0412191, to appear in Commun. Math. Phys.

[5] H. Boos and V. Korepin, Quantum spin chains and Riemann zeta functions with odd arguments, hep-th/0104008, J. Phys. A 34 (2001) 5311-5316.

[6] H. Boos, V. Korepin and F. Smirnov, Emptiness formation probability and quantum Knizhnik-Zamolodchikov equation, hep-th/0209246 Nucl. Phys. B Vol. 658/3 (2003) $417-439$.

[7] H. Boos, V. Korepin and F. Smirnov, New formulae for solutions of quantum KnizhnikZamolodchikov equations on level -4, hep-th/0304077, J. Phys. A 37 (2004) 323-336.

[8] K. Fabricius and B. McCoy, Functional equations and fusion matrices for the eight vertex model, Publ. RIMS, Kyoto Univ. 40 (2004) 905-932.

[9] K. Fabricius and B. McCoy, New developments in the eight-vertex model II. Chains of odd length, cond-mat/0410113

[10] A. Odesskii and B. Feigin, Sklyanin elliptic algebra, Funt. Anal. and its Appl. 23 (1990) 207-214.

[11] A. Odesskii, Elliptic algebras, Russian Math. Surveys 57 (2002) 1-50.

[12] P. A. Griffith and Harris, Principles of algebraic geometry, A. Wiley Interscience, 1978

[13] F. Göhmann, A. Klümper and A. Seel, Integral representation of the density matrix of the XXZ chain at finite temperatures, J.Phys. A38 (2005) 1833-1842 ; cond-mat/0412062

[14] A. Erdélyi et al., Higher transcendental functions, McGraw-Hill, 1953.

[15] M. Jimbo and T. Miwa, Quantum Knizhnik-Zamolodchikov equation at $|q|=1$ and correlation functions of the XXZ model in the gapless regime, J. Phys. A 29 (1996) $2923-2958$

[16] M. Jimbo, T. Miwa and A. Nakayashiki, Difference equations for the correlation functions of the eight vertex model, J. Phys. A 26 (1993), 2199-2209.

[17] M. Jimbo, T. Miwa, K. Miki and A. Nakayashiki, Correlation functions of the XXZ model for $\Delta<-1$, Phys. Lett. A 168 (1992), 256-263.

[18] N. Kitanine, J. M. Maillet, N. A. Slavnov and V. Terras, Master equation for spin-spin correlation functions of the XXZ chain, hep-th/0406190

[19] N. Kitanine, J. M. Maillet, N. A. Slavnov and V. Terras, Dynamical correlation functions of the XXZ spin-1/2 chain, hep-th/0407223

[20] N. Kitanine, J.-M. Maillet and V. Terras, Correlation functions of the XXZ Heisenberg spin- $\frac{1}{2}$-chain in a magnetic field, Nucl. Phys. B 567 (2000), 554-582.

[21] C. Korff, Auxiliary matrices on both sides of the equator, J.Phys.A38 (2005), 47-66.

[22] C. Korff, A Q-operator identity for the correlation functions of the infinite XXZ spinchain, hep-th/0503130

[23] G. Kato, M. Shiroishi, M. Takahashi, K. Sakai, Third-neighbor and other four-point correlation functions of spin-1/2 XXZ chain, J. Phys. A: Math. Gen. 37 (2004) 5097.

[24] S. Lukyanov and Y. Pugai, Multi-point local height probabilities in the integrable RSOS model, Nucl.Phys. B473[FS] (1996) 631-658.

[25] M. Lashkevich and Y. Pugai, Free field construction for correlation functions of the eight-vertex model, Nucl.Phys. B516 (1998) 623-651.

[26] M. Lashkevich and Y. Pugai, Nearest neighbor two-point correlation function of the $Z$-invariant eight-vertex model, JETP Lett. 68 (1998) 257-262.

[27] A. Pronko and Yu. Stroganov, Bethe equations "on the wrong side of equator, J. Phys. A32 (1999), 2333-2340 
[28] S. N. M. Ruijsenaars, First order analytic difference equations and integrable quantum systems, J. Math. Phys.38 (1997) 1069-1146

[29] K. Saito, Einfach-elliptische Singularitäten, Inventiones math. 23 (1974), 289-325.

[30] K. Saito, Period mapping associated to a primitive form, Publ. RIMS, Kyoto Univ.19 (1983), 1231-1264.

[31] K. Saito, Extended affine root systems I (Coxeter transformations), Publ. RIMS, Kyoto Univ.21 (1985), 75-179.

[32] K. Saito, On the periods of primitive integrals I, RIMS preprint 412 (1982).

[33] J. Shiraishi, Free field constructions for the elliptic algebra $\mathcal{A}_{q, p}\left(\hat{s} l_{2}\right)$ and Baxter's eight-vertex model, Int. J. Mod. Phys. A19 (2004) 363-380; math.QA/0302097.

[34] J. Shiraishi, A commutative family of integral transformations and basic hypergeometric series I,II, math.QA/0501251, 0502228.

[35] E. K. Sklyanin, Some algebraic structures connected with the Yang-Baxter equation, Func. Anal. and Appl. 16 (1982) 27-34.

[36] E. K. Sklyanin, Some algebraic structures connected with the Yang-Baxter equation. Representations of quantum algebras, Func. Anal. and Appl. 17 (1983) 34-48.

[37] M. Takahashi, G. Kato, M. Shiroishi, Next nearest-neighbor correlation functions of the spin-1/2 XXZ chain at massive region, J. Phys. Soc. Jpn. 73 (2004) 245.

HB: Physics Department, University of Wuppertal, D-42097, Wuppertal, GerMANY $^{4}$

E-mail address: boos@physik.uni-wuppertal.de

MJ: Graduate School of Mathematical Sciences, The University of Tokyo, Tokyo 153-8914, JAPAN

E-mail address: jimbomic@ms.u-tokyo.ac.jp

TM: Department of Mathematics, Graduate School of Science, Kyoto UniverSITY, КYOTO 606-8502, JAPAN

E-mail address: tetsuji@math.kyoto-u.ac.jp

FS $^{5}$ : Laboratoire de Physique Théorique et Hautes Energies, Université Pierre et Marie Curie, Tour $161^{\mathrm{er}}$ Étage, 4 Place Jussieu 75252 Paris Cedex 05, France

E-mail address: smirnov@lpthe.jussieu.fr

YT: Graduate School of Pure and Applied Sciences, Tsukuba University, Tsukuba, IBARAKI 305-8571, JAPAN

E-mail address: takeyama@math.tsukuba.ac.jp

\footnotetext{
${ }^{4}$ on leave of absence from the Institute for High Energy Physics, Protvino, 142281, Russia

${ }^{5}$ Membre du CNRS
} 